\title{
Sonic Hedgehog Signaling Controls Thalamic Progenitor Identity and Nuclei Specification in Mice
}

\author{
Tou Yia Vue, ${ }^{1,2,3}$ Krista Bluske, ${ }^{1,2,3}$ Amin Alishahi, ${ }^{1,2}$ Lin Lin Yang, ${ }^{4}$ Naoko Koyano-Nakagawa, ${ }^{1,2,3}$ Bennett Novitch, ${ }^{4}$ and \\ Yasushi Nakagawa ${ }^{1,2,3}$ \\ ${ }^{1}$ Department of Neuroscience and ${ }^{2}$ Stem Cell Institute, University of Minnesota Medical School, and ${ }^{3}$ Graduate Program in Neuroscience, University of \\ Minnesota, Minneapolis, Minnesota 55455, and ${ }^{4}$ Department of Neurobiology, David Geffen School of Medicine, University of California, Los Angeles, Los \\ Angeles, California 90095
}

The mammalian thalamus is located in the diencephalon and is composed of dozens of morphologically and functionally distinct nuclei. The majority of these nuclei project axons to the neocortex in unique patterns and play critical roles in sensory, motor, and cognitive functions. It has been assumed that the adult thalamus is derived from neural progenitor cells located within the alar plate of the caudal diencephalon. Nevertheless, how a distinct array of postmitotic thalamic nuclei emerge from this single developmental unit has remained largely unknown. Our recent studies found that these thalamic nuclei are in fact derived from molecularly heterogeneous populations of progenitor cells distributed within at least two distinct progenitor domains in the caudal diencephalon. In this study, we investigated how such molecular heterogeneity is established and maintained during early development of the thalamus and how early signaling mechanisms influence the formation of postmitotic thalamic nuclei. By using mouse genetics and in utero electroporation, we provide evidence that Sonic hedgehog $(\mathrm{Shh})$, which is normally expressed in ventral and rostral borders of the embryonic thalamus, plays a crucial role in patterning progenitor domains throughout the thalamus. We also show that increasing or decreasing Shh activity causes dramatic reorganization of postmitotic thalamic nuclei through altering the positional identity of progenitor cells.

\section{Introduction}

The thalamus is located in the vertebrate diencephalon and plays critical roles in controlling animal behavior. Each of the thalamic nuclei exhibits a unique pattern of gene expression and connectivity. In mammals, a vast majority of thalamic nuclei send axons to unique sets of distinct neocortical areas. For example, principal sensory nuclei (visual, somatosensory, and auditory) establish topographic and area-specific thalamocortical projections to primary sensory areas in the neocortex (Jones, 2007). Despite the functional importance of the thalamus, however, molecular mechanisms that control the specification of thalamic nuclei are not well understood. A particularly intriguing question is how a single developmental unit in the alar plate of caudal diencephalon [prosomere 2 in the study by Puelles and Rubenstein (2003)] can

\footnotetext{
Received Feb. 6, 2009; revised March 6, 2009; accepted March 9, 2009.

This work was supported by Minnesota Medical Foundation, Grant-in-Aid Program of University of Minnesota Graduate School, National Institute of Neurological Disorders and Stroke Grant R01 NS049357, and Whitehall Foundation. We thank Aya Taniguchi, Anu Jayabalu, Christina Kazemzadeh, Lauren Bolopue, and Melody Lee for help in gene targeting, maintaining mouse colonies, and genotyping embryos; Yasuhiko Kawakami and members of the Nakagawa and Koyano laboratories for discussion; James Briscoe, Martyn Goulding, Peter Gruss, Gail Martin, Andrew McMahon, and Masato Nakafuku for plasmids; Andras Nagy for R1 ES cells; Jun-ichi Miyazaki for CAG promoter; Tomomi Shimogori and Tetsuichiro Saito for advice on in utero electroporation; Kyuson Yun for Nestin ${ }^{\mathrm{Cre} /+}$ mice; and

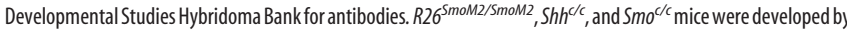
Andrew McMahon's laboratory and were obtained from The Jackson Laboratory. University of Minnesota Mouse Genetics Facility provided neomycin-resistant fibroblasts for ES cell feeders, and Thom Sanders, Elizabeth Hughes, and Tina Jones and University of Michigan Transgenic Animal Model Core contributed to the production of Olig3 $3^{\text {Cre }}$ chimeras. We thank Paul Letourneau and Steve McLoon for critical reading of early versions of the manuscript and Masato Nakafuku for encouragement and helpful discussion.

Correspondence should be addressed to Yasushi Nakagawa at the above address. E-mail: nakagawa@umn.edu. DOI:10.1523/JNEUROSCI.0656-09.2009

Copyright $\odot 2009$ Society for Neuroscience $\quad 0270-6474 / 09 / 294484-14 \$ 15.00 / 0$
}

generate such a diverse array of postmitotic nuclei at later stages of development.

Numerous studies have shown that ventral-to-dorsal gradient of Shh activity specifies different neuronal subtypes throughout the embryonic CNS (Fuccillo et al., 2006). In the diencephalon, Shh is not only expressed in the basal plate, which is ventral to the thalamus, but is also expressed in the zona limitans intrathalamica (ZLI), a dorsally extending group of cells located immediately rostral to the early embryonic thalamus (Puelles and Rubenstein, 1993; Shimamura et al., 1995). In ovo electroporation and grafting studies in chick showed that ectopic expression of Shh in the caudal diencephalon and mesencephalon induces the expression of Gbx2 and reduces Pax6 (Kiecker and Lumsden, 2004; Vieira et al., 2005). Conversely, inhibition of Shh signaling by a dominant-negative form of Shh receptor Ptc1 reduced Nkx2.2, Ptc1, and Gbx2 expression (Kiecker and Lumsden, 2004). Together, these studies establish the importance of Shh signaling in the global regionalization of the diencephalon, particularly its role in specifying the identity of the thalamus as a whole. To date, however, whether Shh signaling also controls the identity of progenitor cells within the thalamus along the rostrocaudal axis and subsequently contributes to the specification of different thalamic nuclei has remained largely unknown.

We recently reported molecular heterogeneity of thalamic progenitor cells in the mouse and analyzed the postmitotic fate of each progenitor cell population using genetic lineage tracing methods (Vue et al., 2007). We proposed that the thalamic ventricular zone is marked by the expression of the basic-helix-loophelix (bHLH) transcription factor Olig3, and is divided into two 
A

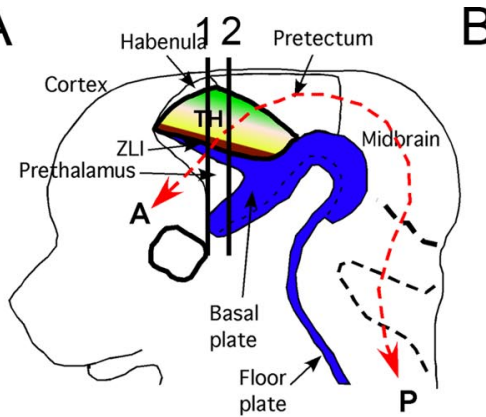

B

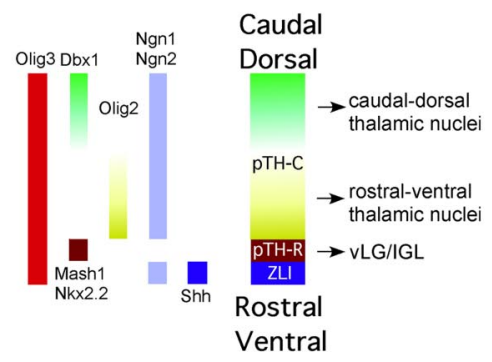

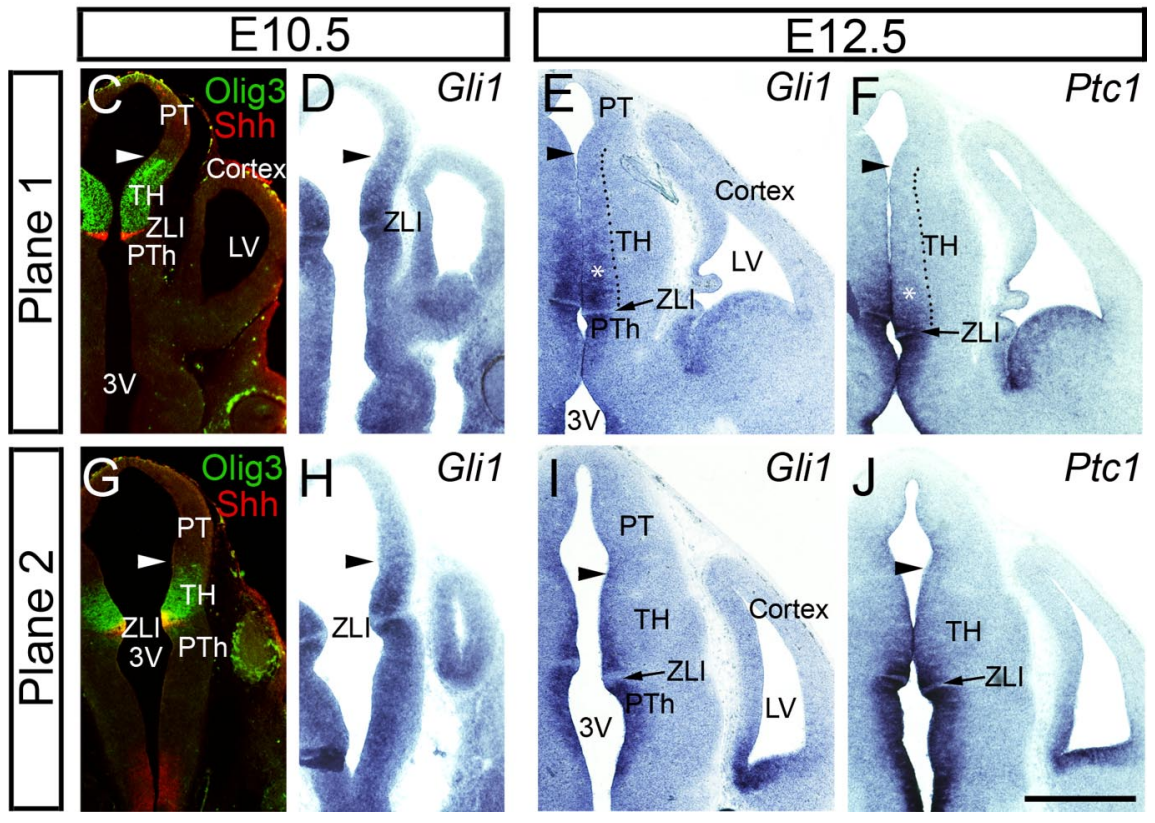

Figure 1. Thalamic progenitor cells are exposed to graded activity of Shh signaling. $\boldsymbol{A}, \mathrm{A}$ schematic of the location of the thalamus (TH) in the caudal diencephalon of the mouse embryo. Embryo is facing left. The thalamus is bordered by the pretectum caudally, the habenula dorsally, the basal plate ventrally, and by the ZLI rostrally. The basal plate and ZLI are sources of Shh (blue). Red dashed line shows the curvature of the anterior-posterior (AP) axis of the diencephalon. The two vertical lines (1 and 2) represent the planes of frontal sections shown in $\boldsymbol{C}-\boldsymbol{J}$. $\boldsymbol{B}$, Expression patterns of bHLH (0lig2, 0lig3, Ngn1/2, Mash1) and homeodomain (Nkx2.2, Dbx1) transcription factors in the thalamic ventricular zone. Olig3 is expressed in the entire thalamic ventricular zone and the ZLI. Expression of Mash1, Nkx2.2, Ngn1, and Ngn2 divides the thalamus into two distinct progenitor domains, a small rostral domain (pTH-R) and a much larger caudal domain (pTH-C). Olig2 and Dbx1 are expressed in opposing gradients within pTH-C. $(-J$, Graded patterns of Gli1 and Ptc1 expression in thalamic progenitor cells. Frontal sections through the thalamus of E10.5 $(\boldsymbol{C}, \boldsymbol{D}, \boldsymbol{G}, \boldsymbol{H})$ and E12.5 $(\boldsymbol{E}, \boldsymbol{F}, \boldsymbol{I}, \boldsymbol{J})$ wild-type brains. Top panels $(\boldsymbol{C}-\boldsymbol{F})$ are sections at a more dorsal and rostral level (plane 1 in $\boldsymbol{A}$ ) and the bottom panels $(\boldsymbol{G} \boldsymbol{J})$ are for a more ventral and caudal level (plane 2 in $\boldsymbol{A}$ ), showing similar results. $\boldsymbol{C}, \boldsymbol{G}$, Double immunofluorescence for 0 lig3 and Shh atE10.5 to show the location of the thalamus. Olig3 expression caudal to the ZLI marks the entire thalamic ventricular zone. $\boldsymbol{D}, \boldsymbol{H}$, In situ hybridization for Gli1 on sections adjacent to $\boldsymbol{C}$ and $\mathbf{G}$. Gli1 expression is higher closer to the ZLI and decreases in a graded manner throughout the thalamic ventricular zone. Arrowheads indicate dorsal and caudal boundary of the thalamus delineated by 0 lig3 expression. $\boldsymbol{E}, \mathbf{I}$, In situ hybridization for Gli1 at E12.5, showing a similar gradient to E10.5. $\boldsymbol{F}, \boldsymbol{J}$, In situ hybridization for Ptc 1 on adjacent sections to $\boldsymbol{E}$ and $\boldsymbol{I}$, respectively, again showing similar gradients. Asterisk and dotted lines in $\boldsymbol{E}$ and $\boldsymbol{F}$ mark the thalamic ventricular zone, indicating that expression of Gli1 and Ptc 1 is restricted to progenitor cells. 3V, Third ventricle; LV, lateral ventricle; PT, pretectum; TH, thalamus; PTh, prethalamus. Scale bar, $500 \mu \mathrm{m}$.

differentially regulated by Shh in the ventral spinal cord (Briscoe and Novitch, 2008), our previous study predicted that differential Shh signaling may impart not only the specification of PTH-R and pTH-C, but also the molecular heterogeneity of progenitor cells within the $\mathrm{pTH}-\mathrm{C}$ domain. To test this hypothesis, we used conditional gene activation or deletion as well as in utero electroporation in mice to increase or decrease Shh activity in thalamic progenitor cells in temporally and spatially restricted manners. We found that altering the level of Shh activity shifts the positional gene expression patterns in both pTH-R and pTH-C. In addition, this shift results in reorganization of postmitotic thalamic nuclei.

\section{Materials and Methods}

Mice. Care and experimentation on mice were done in accordance with the Institutional Animal Care and Use Committee of the University of Minnesota. Noon of the day on which the vaginal plug was found was counted as embryonic day 0.5 (E0.5). Stages of early embryos were confirmed by morphology (Kaufman, 1992). To generate $\mathrm{Nes} \mathrm{Cre} /+^{\circ}$ R $26^{\mathrm{SmoM} 2 /+} \mathrm{com}-$ pound heterozygous mice, we crossed heterozygous Nestin-Cre $\left(\mathrm{Nes}^{\mathrm{Cre} /+}\right)$ transgenic mice (Tronche et al., 1999) with homozygous ROSA26-stop-SmoM2-EYFP (R26 $\left.6^{\text {SmoM2/SmoM2 }}\right)$ mice (Jeong et al., 2004) and identified Crepositive embryos by PCR. Cre-negative littermates were used as controls. Nes ${ }^{\mathrm{Cr} /+}$; $R 26^{\text {SmoM2/+ }}$ mice died by the end of the first postnatal day (P0). For Shh conditional knockout, we crossed $\mathrm{Nes}^{\mathrm{Cre} /+}$; Shh ${ }^{\mathrm{cl}+}$ mice with $S h h^{c / c}$ mice. $S h h^{c}$ is a conditional allele for $S h h$ (Lewis et al., 2001). PCR was done to identify $\mathrm{Nes}^{\mathrm{Cre}}$ allele as well as floxed and wild-type alleles of Shh. Smo conditional knock-out mice were generated by crossing $\mathrm{Nes}^{\mathrm{Cre} /+} ; \mathrm{Smo}^{\mathrm{cl+}}$ mice with $S m o^{c / c}$ mice (Long et al., 2001). Postmitotic fates of Olig2-expressing progenitor cells were analyzed by crossing Olig $2^{\mathrm{Cre} /+}$ mice (Dessaud et al., 2007) with R26-stop-EGFP reporter mice. Olig2 mutant embryos were obtained by crossing between Olig2 $2^{I R E S-E G F P /+}$ mice (Mukouyama et al., 2006). For analysis of normal gene expression (Fig. 1) and in utero electroporation, timed pregnant CD1 mice (Charles River) were used. For all the other mice, colonies were maintained in $\mathrm{C} 57 \mathrm{~B} / 6 \mathrm{~J}$ background.

Production of Olig3 ${ }^{\mathrm{Cre} /+}$ mice. We produced

distinct progenitor domains, pTH-R and pTH-C (Fig. 1A,B). The pTH-R domain is rostroventrally located within the thalamic primordium, expresses transcription factors Nkx2.2 and Mash1, and contributes largely to nuclei that do not project to the cortex. These nuclei were previously not considered to be part of the postmitotic thalamus. The other domain, pTH-C, expresses Ngn1 and Ngn2 and gives rise to all the classical thalamic nuclei projecting to the cortex. Within $\mathrm{pTH}-\mathrm{C}$, Olig2, a bHLH protein, is expressed in a high-rostroventral to low-caudodorsal gradient, while the homeodomain protein Dbx1 is expressed in the opposite gradient. Because Nkx2.2, Olig2, and Dbx1 are known to be
Olig $3^{\mathrm{Cre} /+}$ mice by homologous recombination in embryonic stem (ES) cells. In short, R1 ES cells were transfected with a targeting vector so that the entire coding region of the Olig3 gene is replaced by a cassette encoding Cre recombinase followed by frt-neo-frt (FNF) sequence (see Fig. $3 K)$. G418-resistant clones were selected and analyzed by Southern blot analysis for homologous recombinants. Two positive clones were microinjected into blastocysts obtained from the mating of C57BL/6NCrl female mice with $(\mathrm{C} 57 \mathrm{BL} / 6 \mathrm{~J} \times \mathrm{DBA} / 2 \mathrm{~J}) \mathrm{F} 1$ male mice (performed at Transgenic Animal Model Core of University of Michigan), and male chimeras were further bred with $\mathrm{C} 57 \mathrm{BL} / 6 \mathrm{~J}$ females to produce heterozygous Olig3 $3^{\mathrm{Cre} /+}$ mice. Olig3 ${ }^{\mathrm{Cre} /+}$ mice were bred with $\mathrm{R}_{2} 6^{\mathrm{SmoM} 2 / \mathrm{SmoM} 2}$ 
mice to obtain $\mathrm{Olig} 3^{\mathrm{Cre} /+} ; \mathrm{R}^{\mathrm{SmoM} 2 /+}$ mice. To produce conditional $\mathrm{Smo}$ knock-out mice, we crossed $\mathrm{Olig}^{\mathrm{Cre} /+} ; \mathrm{Smo}^{c /+}$ and $\mathrm{Smo} o^{c / c}$ mice. To monitor the spatial and temporal patterns of Cre-mediated recombination, $\mathrm{Nes}{ }^{\mathrm{Cre} /+}$ or Olig $3^{\mathrm{Cre} /+}$ mice were bred with R26-stop-EYFP reporter mice (Srinivas et al., 2001), and embryos were analyzed for the expression of EYFP and various progenitor cell markers.

In utero electroporation. In utero electroporation was performed as described previously (Saito and Nakatsuji, 2001). Timed pregnant (E10.5 or E11.5) CD1 females were anesthetized by intraperitoneal injection of pentobarbital sodium ( $2.0 \mathrm{mg}$ for $30 \mathrm{~g}$ dams). Embryos were visualized by illumination of the uterus with fiber optics, and a pulled micropipette (1B120F-6, World Precision Instruments) loaded with DNA was inserted into the third ventricle of each embryo. Approximately $0.5 \mu \mathrm{l}$ of the DNA solution was injected with Picospritzer III (Parker Instruments). Four pulses of square-wave current were applied ( $35 \mathrm{~V}, 30 \mathrm{~ms}$ on and $100 \mathrm{~ms}$ off), using CUY21EDIT electroporator (NEPAGENE) and paddle- or needle-type electrodes. We used $4 \mu \mathrm{g} / \mu \mathrm{l} p C A G$-Olig2 (from Masato Nakafuku, Cincinnati Children's Hospital Medical Center, Cincinnati, $\mathrm{OH})$ plus $0.5 \mu \mathrm{g} / \mu \mathrm{l}$ pCAG-nuclear EGFP and $2 \mu \mathrm{g} / \mu \mathrm{l} p C A G$-SmoM2-EYFP (from Andrew McMahon, Harvard University, Cambridge, MA). After the injection, the dam was sutured and allowed to recover until analysis.

In situ hybridization and immunohistochemistry. In situ hybridization and immunohistochemistry were done based on Vue et al. (2007). In this study, goat anti-Sox1 antibody from R\&D Systems was used at 1:100. cDNAs for BHLHB4, Gli1, Ptc1, Dlx2, Sox14, and Pdlim3 were obtained from Open Biosystems. Irx2 and Spry1/Spry2 cDNAs were obtained from Peter Gruss (Max Planck Society, Munich, Germany) and Gail Martin (University of California, San Francisco, San Francisco, CA), respectively. Sox1-positive cells in the intergeniculate leaflet (IGL) nucleus were counted and compared between $\mathrm{Olig}^{\mathrm{Cre} /+} ; \mathrm{Smo}^{c / c}$ and control embryos. Serial sections from each brain were collected onto 8 slides, and Sox1positive cells within NPY-expressing IGL nucleus were counted with ImageJ software. Average cell counts per slide were compared between the two genotypes ( 4 thalami in each group), and Student's $t$ test was performed to test the significance of difference. An SEM was used for presentation of data.

Analysis of cell proliferation in the thalamic ventricular zone of SmoM2expressing or Shh knock-out brains. Brains of E11.5 Olig $3^{\mathrm{Cre} /+}$; R26 $6^{\text {SmoM2/+ }}$ and control litter mates, or E12.5 Nes ${ }^{\mathrm{Cre} /+}$; Shh ${ }^{c / c}$ and control littermates were cryosectioned at $20 \mu \mathrm{m}$ thickness and then immunostained with anti-phospho histone $\mathrm{H} 3$ (anti-PH3) antibody (1:100, Millipore \#06-570) to label cells in M-phase. PH3-positive cells situated caudal to the ZLI and within the Olig3-expressing domain in the diencephalon were manually counted, and the numbers were compared between $\mathrm{Olig} 3^{\mathrm{Cre} /+} ; \mathrm{R}_{2} 6^{\mathrm{SmoM} 2 /+}$ or $\mathrm{Nes}{ }^{\mathrm{Cre} /+}$; $\mathrm{Shh}^{\text {c/c }}$ with their respective controls. Student's $t$ test was performed to test the significance of difference, and an SEM was used for presentation of data.

Anterograde labeling of retinogeniculate axons and their targets in the thalamus. Approximately $2 \mu \mathrm{l}$ of cholera toxin B subunit [CTB (5 $\mu \mathrm{g} / \mu \mathrm{l})$, List Biological Laboratories or Invitrogen] was injected into the right retina of neonatal $\mathrm{Olig} 3^{\mathrm{Cre} /+} ; \mathrm{R}^{\mathrm{SmoM} 2 /+}$ mice and their control littermates. After an overnight survival period, pups were perfused with $4 \%$ paraformaldehyde and processed for cryosectioning. Goat anti-CTB antibody (1:100, List Biological Laboratories) was used to detect the retinal ganglion cell axons as well as their targets in the contralateral diencephalon, the dorsal lateral geniculate (dLG) and ventral lateral geniculate (vLG) nuclei as well as IGL. We compared the areas of terminations by measuring the dLG between Cre+ pups and $\mathrm{Cre}-$ controls. The border between $\mathrm{dLG}$ and IGL was identified by DAPI staining and immunohistochemistry with anti-Sox2. Scanned images were analyzed by ImageJ software for area measurement. We measured the area of every section containing dLG (21-29 sections per brain, $20 \mu \mathrm{m}$ thickness) and calculated the estimated volume of dLG by summing the values of area $\times$ thickness of each section. Student's $t$ test was performed to test the significance of difference, and an SEM was used for presentation of data.

\section{Results}

Shh signaling is graded within the thalamic progenitor zone

Within the diencephalon, Shh is expressed in the ZLI and the basal plate, which directly border the thalamus rostrally and ventrally, respectively (Fig. $1 A$ ). In this study we asked whether differential Shh signaling is responsible for setting up the expression patterns of the various transcription factors that we described previously (Fig. $1 B$ ) and later contributes to the specification of different thalamic nuclei. To determine whether the embryonic thalamus is indeed exposed to graded Shh activity, we analyzed the expression of Gli1 and Ptc1 in mouse embryos from E10.5 to E12.5. Gli1 and Ptc1 are direct target genes of the Shh transduction pathway, and their expression levels reflect the levels of Shh activity in the cell (Agren et al., 2004; Bai et al., 2004). We predicted that if thalamic progenitor cells are differentially patterned by Shh signaling, then both Gli1 and Ptcl should be expressed in graded manners across pTH-R and pTH-C. Indeed, at both E10.5 and E12.5, Gli1 and Ptc1 were expressed in a highrostroventral to low-caudodorsal gradient in wild-type brains (Fig. $1 D-F, H-J$; thalamus is defined by Shh and Olig3 in $C$ and $G)$; both genes were expressed at the highest levels in pTH-R, and their expression gradually tapered off caudally and dorsally away from the ZLI and the basal plate. With this finding, we hypothesized that the highest level of Shh signaling defines pTH-R, and progressively lower levels define different positional identity of progenitor cells within pTH-C depending on the distance from the ZLI and the basal plate. It was also evident that expression of Gli1 and Ptc1 is found only in progenitor cells of the thalamic ventricular zone (Fig. $1 E, F, I, J$ ) and not in postmitotic cells in the mantle zone, demonstrating that Shh signaling, whose direct output is gene activation mediated by Gli transcription factors, is restricted to neural progenitor cells.

\section{Ectopic enhancement of Shh signaling strongly induces Gli1 and Ptc1 in the entire thalamic progenitor domains}

Based on the above findings, we examined whether increased Shh activity in thalamic progenitor cells can cause a rostroventral shift in their positional identity. More specifically, we predicted that if caudodorsal progenitors are exposed to a higher level of Shh activity than normal, these progenitor cells would express rostroventral molecular markers rather than caudodorsal ones. To test this possibility, we crossed NestinCre $\left(\mathrm{Nes}^{\mathrm{Cre} /+}\right)$ transgenic mice (Tronche et al., 1999) with ROSA26-stop-SmoM2-EYFP (R26 $\left.6^{\text {SmoM2/SmoM2 }}\right)$ mice (Jeong et al., 2004) to express the fusion protein of SmoM2-EYFP broadly in neural progenitor cells, including those in the thalamus. SmoM2 carries a missense mutation in the Smoothened (Smo) gene, which encodes a transmembrane Shh effector. SmoM2-expressing cells undergo cell-autonomous activation of Shh signaling independent of ligand binding (Xie et al., 1998). Thus, we expected that mis-expression of SmoM2 would cause elevated Shh signaling in all thalamic progenitor cells. By comparing $\mathrm{Nes}^{\mathrm{Cre} /+} ; \mathrm{R} 26^{\mathrm{SmoM} 2 /+}$ and Cre-negative, $R 26^{\text {SmoM2/+ }}$ controls and wild-type embryos, we found that Cre-negative control and wild-type embryos show the same gene expression and morphology at all stages. In contrast, brains of $\mathrm{NeS}^{\mathrm{Cre} /+}$; $\mathrm{R}_{2} 6^{\mathrm{SmoM} 2 /+}$ mice were larger in size than Cre-negative littermates, especially in the dorsal telencephalon.

Analysis of SmoM2-EYFP expression as detected by antiEGFP antibody showed that in $\mathrm{NeS}^{\mathrm{Cre} /+} ; \mathrm{R}_{2} 6^{\mathrm{SmoM} 2 /+}$ embryos, 


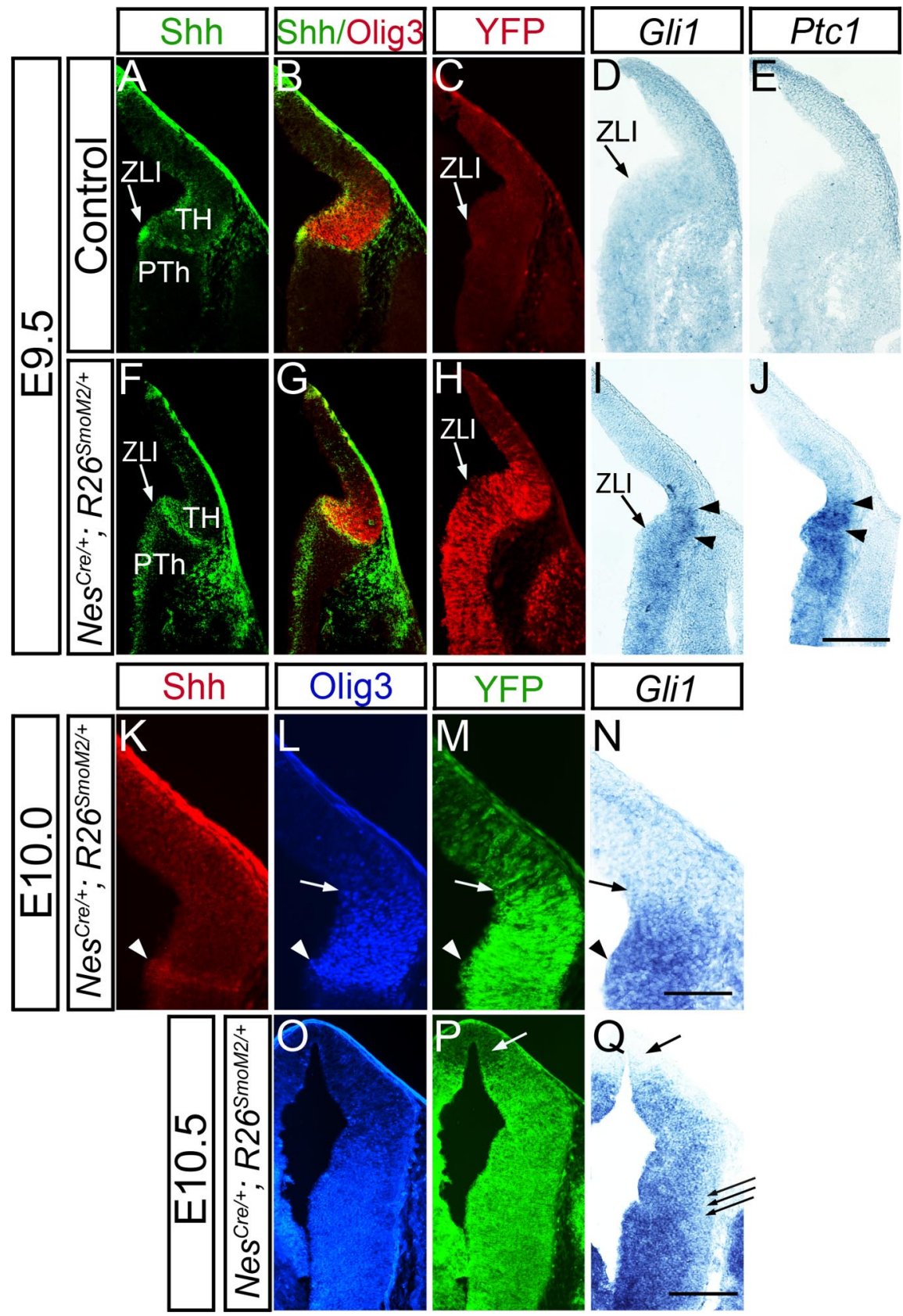

Figure 2. Ectopic expression of $\mathrm{SmoM} 2$ in neural progenitor cells elevates the level of Shh signaling throughout the entire thalamic ventricular zone. $\boldsymbol{A}-\boldsymbol{Q}$, Frontal sections of $\mathrm{Nes}^{\mathrm{Cre} /+} ; R_{2} 6^{\text {SmoM2/+ }}(\boldsymbol{F}-\boldsymbol{Q})$ and $\mathrm{Cre}-\operatorname{control}(\boldsymbol{A}-\boldsymbol{E})$ embryos at E9.5 $(\boldsymbol{A}-\boldsymbol{J})$, E10.0 $(\boldsymbol{K}-\boldsymbol{N})$, and E10.5 (O-Q). Midline is to the left. $\boldsymbol{A}-\boldsymbol{J}$, Controls $(\boldsymbol{A}-\boldsymbol{E})$ are compared with Nes ${ }^{\mathrm{Cre} /{ }^{+}} ; R 26^{\text {SmoM2/+}}$ littermates $(\boldsymbol{F}-\boldsymbol{J})$ at $E 9.5 . A, B, F, G$, Double immunofluorescence for Shh and 0 lig3 to show the locations of the $Z L I$ and thalamus, which are similar between control and $\mathrm{NeS}^{\mathrm{Cre} /+} ; \mathrm{R}_{2} 6^{\text {SmoM2/+ }}$ embryos. EYFP immunofluorescence shows that Cre-mediated misexpression of SmoM2-EYFP in the thalamus starts to occur at E9.5, but only in the vicinity of the ZLI ( $\boldsymbol{C}, \boldsymbol{H}$, arrowheads). $\boldsymbol{C}$ and $\boldsymbol{H}$ are adjacent to $\boldsymbol{B}$ and $\mathbf{G}$, respectively. In situ hybridization analysis for $G$ lil and Ptc 1 at E9.5 $(\boldsymbol{D}, \boldsymbol{E}, \boldsymbol{I}, \boldsymbol{J})$ shows that $G$ li 1 and Ptc 1 are not yet expressed in Cre - controls $(\boldsymbol{D}, \boldsymbol{E})$, but in SmoM2-expressing brain, both Gli1 and Ptc1 are prematurely induced in the thalamus (I, $\boldsymbol{J}$, arrowheads) as well as in the prethalamus. $\boldsymbol{K}-\boldsymbol{N}, \mathrm{In} \mathrm{Nes}^{\mathrm{Cre} /+} ; R 26^{\text {SmoM2/+ }}$ embryos at E10.0, recombination as indicated by SmoM2-YFP expression spans the entire thalamus marked by 0 lig3 expression. Gli1 expression follows this. Arrowhead indicates the ZLI, and arrow shows the caudodorsal border of the thalamus. $\mathbf{O} \mathbf{Q}, \mathrm{In} \mathrm{Nes}^{\mathrm{Cre} /{ }^{+}} ; \mathrm{R}_{2} 6^{\mathrm{SmoM} 2 /+}$ embryos at E10.5, recombination has further progressed, and SmoM2-EYFP expression is found in most of the diencephalon. The arrow indicates the most caudaldorsal part of the diencephalon that has not yet undergone recombination. Triple arrows show that the mantle zone of the thalamus does not express Gli1. Scale bar, $200 \mu \mathrm{m}$ for $\mathbf{A}-\mathbf{N}, 500 \mu \mathrm{m}$ for $\mathbf{0} \mathbf{Q}$.

Cre-mediated recombination starts to occur in the diencephalon by E9.5 (Fig. $2 A-C, F-H$ ). At this stage, Shh expression in the ZLI has just started (Fig. $2 A, F$ ), and Gli1 and Ptc1 were not clearly detectable in control embryos (Fig. $2 D, E$ ). However, in
Nes ${ }^{\mathrm{Cre} /+} ; \mathrm{R}^{\mathrm{SmoM} 2 /+}$ embryos, Gli1 and Ptc1 were already expressed ectopically in thalamic progenitor cells near the ZLI (Fig. 2I,J). Subsequently, recombination progresses through the thalamus into the more caudodorsal region, and by E10.0, SmoM2-EYFP and Gli1 expression covered the entire thalamus labeled by Olig3 (Fig. $2 K, L, M, N$ ). By E10.5, most embryos we analyzed showed broad expression of SmoM2EYFP and Gli1 in the entire diencephalon except the most dorsal portion (Fig. $2 P, Q)$. Analysis of recombination using R26-stop-EYFP reporter mice showed a similar rostroventral to caudodorsal progression of EYFP expression (supplemental Fig. $S 1 A-D$, available at www. jneurosci.org as supplemental material). These results demonstrate that in Nes ${ }^{\mathrm{Cre} /+}$; $\mathrm{R}_{2} 6^{\text {SmoM2/+ }}$ embryos, Shh signaling is elevated in a rostroventral to caudodorsal direction, and by E10.0, there is a uniform enhancement in signaling within the entire thalamic ventricular zone.

\section{Increased Shh activity induces rostroventral markers in thalamic progenitor cells}

We then asked whether the increased Shh signaling in $\mathrm{Nes}^{\mathrm{Cre} /+} ; \mathrm{R}^{\mathrm{SmoM} 2 /+}$ embryos alters the expression profile of transcription factors in the thalamus. In $\mathrm{Nes}^{\mathrm{Cre} /+}$; $R 26^{\text {SmoM2/+ }}$ embryos, expression of Shh in the ZLI was not affected (Fig. $3 A, B, E, F$ ). Nonetheless, expression of $\mathrm{Nkx} 2.2$ and Mash1, two markers for the rostral progenitor domain, pTH-R, was expanded (Fig. $3 C, D, G, H$ ). This expansion results in more than two-fold increase in the size of pTH-R and disrupts the normally distinct gene expression boundary between pTH-R and pTH-C. The once distinct boundary is now replaced by a "mixed zone (pTH-R/C)" in which some cells expressing only pTH-R markers (Nkx2.2 and Mash1) are intermingled with cells expressing only pTH-C markers (Ngn2 and Olig2) (Fig. 3G,H). Such a zone of mixed pTH-R and pTH-C identity was not seen in control embryos (Fig. 3C,D). Although expression of SmoM2-EYFP appeared ubiquitous and homogeneous throughout the thalamic ventricular zone of $\mathrm{Nes}^{\mathrm{Cre} /+}$; R26 $6^{\text {SmoM2/+ }}$ embryos at E11.5 (supplemental Fig. S1E-H, available at www. jneurosci.org as supplemental material), it is possible that only a small number of cells in this zone expressed ectopic SmoM2 early enough to take on the pTH-R fate, whereas the remaining cells were irreversibly determined to become pTH-C progenitor cells.

SmoM2 acts cell autonomously to enhance Shh signaling (Xie 
et al., 1998). Thus, the observed changes in marker expression are likely to be caused by intrinsic changes in thalamic patterning. Nonetheless, since most CNS progenitor cells are forced to express SmoM2 in $\mathrm{Nes}^{\mathrm{Cre} /+}$; $\mathrm{R}_{2} 6^{\mathrm{SmoM} 2 /+}$ embryos, it is possible that some secondary, indirect effects from outside the thalamus may have contributed to the expansion of pTH-R and/or the formation of the "mixed zone." To investigate this possibility, we took two additional approaches to further restrict the SmoM2 expression spatially and temporally within the thalamus.

First, we performed in utero electroporation of SmoM2 plasmid ( $p C A G-$ SmoM2-EYFP) at E11.5 and analyzed the embryos a day later. We found that similar to $\mathrm{Nes}{ }^{\mathrm{Cre} /+} ; \mathrm{R}_{2} 6^{\mathrm{SmoM} 2 /+}$ embryos, both Nkx2.2 (Fig. 3I,J) and Mash1 (data not shown) are expanded and are ectopically expressed more caudally on the electroporated side compared with the control side. As a second approach, we generated Olig $3^{\mathrm{Cre}}$ mice (Fig. $3 \mathrm{~K}$ ), so that we can express Cre recombinase in pTH-R, pTH-C, and the ZLI, but not in surrounding regions of the forebrain such as the prethalamus, the habenula, the pretectum, the diencephalic basal plate or the telencephalon. Cre-mediated recombination as assessed with R26-stop-EYFP reporter mice (Fig. 3L-O) and ROSA26-stopSmoM2-EYFP mice (supplemental Fig. S1 $I, J$, available at www.jneurosci.org as supplemental material) faithfully followed the expression of the endogenous Olig3 gene. Thus, $\mathrm{Olig}^{\mathrm{Cre}}$ mice are useful in thalamus-specific gene manipulation. We found that brains of $\mathrm{Olig}^{\mathrm{Cre} /+}$; $R 26^{\text {SmoM2/+ }}$ are morphologically indistinguishable from control littermates, and some of these pups survive postnatally. Neuronal differentiation as determined by the expression of TuJ1, a neuronal marker, was also not affected in these embryos (supplemental Fig. S2A,B, available at www.jneurosci.org as supplemental material). Additionally, analysis of cell proliferation in $\mathrm{Olig}^{\mathrm{Cre} /+} ; \mathrm{R}^{\mathrm{SmoM} 2 /+}$ embryos at E11.5 revealed that BrdU-incorporation and phospho-histone $\mathrm{H} 3$ ( $\mathrm{PH} 3$ ), an M-phase marker, also appeared normal (supplemental Fig. S2A,B, available at www. jneurosci.org as supplemental material). Quantification of $\mathrm{PH} 3-$ positive cells in Olig3-expressing thalamic progenitor cells showed that there was no significant difference in number between Olig3 ${ }^{\mathrm{Cre} /+}$; $226^{\text {SmoM2/+ }}$ and control embryos (4440 \pm 206.0, $n=2$ for Olig $3^{\mathrm{Cre} /+} ; \mathrm{R}^{\text {SmoM } 2 /+}, 4906 \pm 449.5, n=2$ for Cre- controls; $p=0.45$ ).

Analysis of progenitor markers in $\mathrm{Olig}_{3}{ }^{\mathrm{Cre} /+} ; \mathrm{R}_{2} 6^{\mathrm{SmoM} 2 /+} \mathrm{em}-$ bryos showed similar changes in gene expression compared with $\mathrm{NeS}^{\mathrm{Cre} /+}$; $\mathrm{R}_{2} 6^{\mathrm{SmoM} 2 /+}$ embryos; pTH-R was expanded, and a "mixed zone (pTH-R/C)" was formed between pTH-C and the

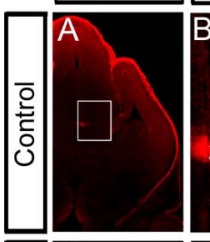

K Olig3 locus
E12.5

Nkx2.2/Olig2 Ngn2/Mash1
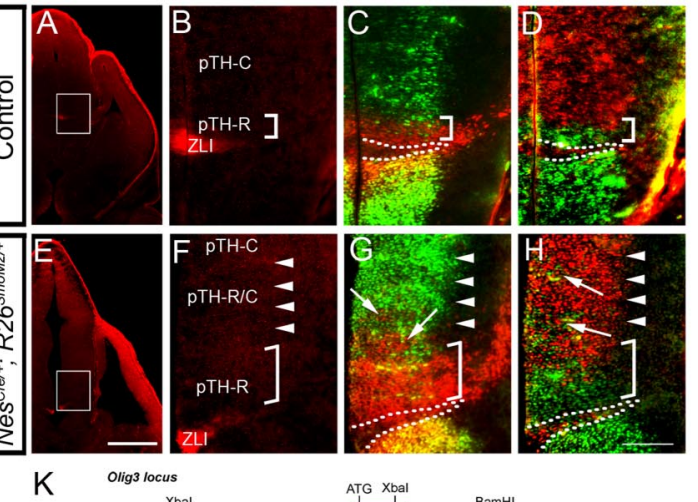

BamHI
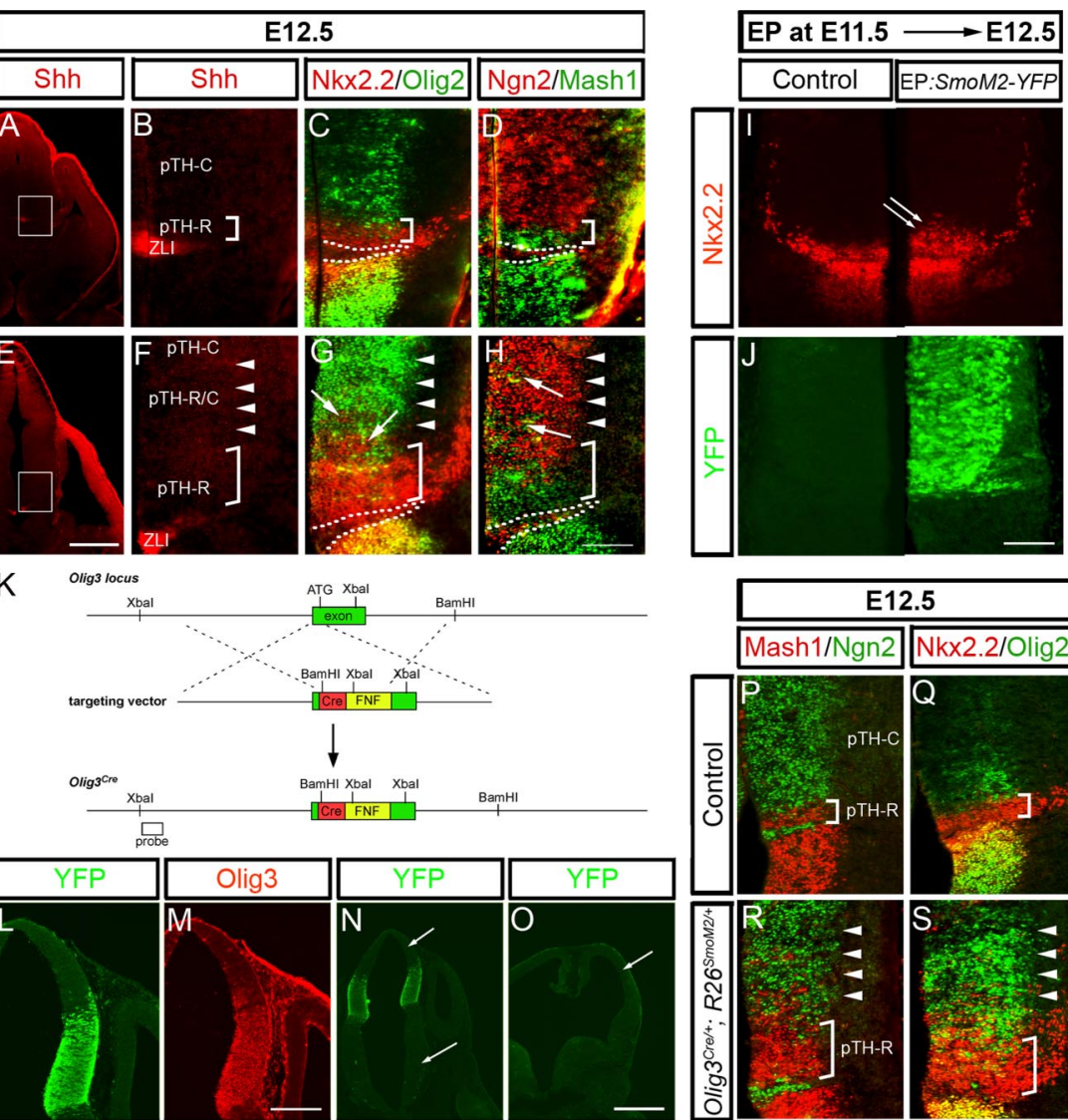

BamHI Xbal Xbal
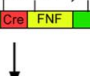

BamHll Xbal Xbal

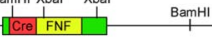
$\stackrel{\text { BamHI }}{\text { I }}$
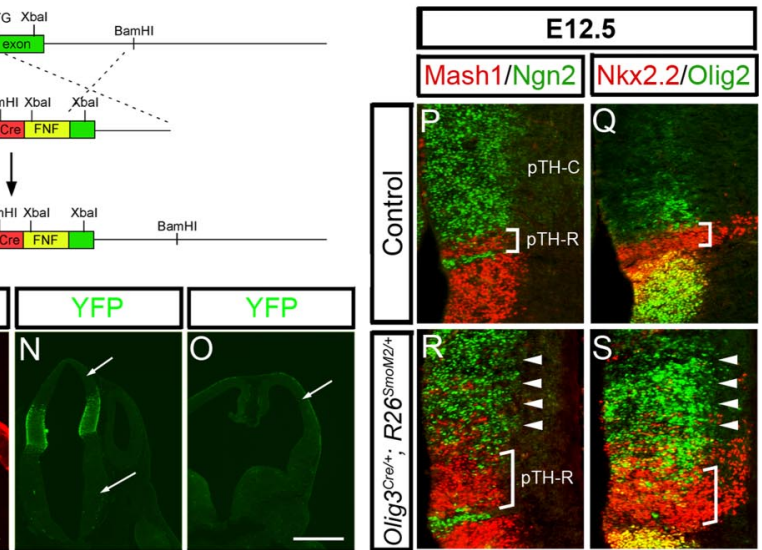

Figure 3. Ectopic SmoM2 expression causes caudodorsal expansion of pTH-R marker genes Nkx2.2 and Mash1 in thalamic progenitors. $\boldsymbol{A}-\boldsymbol{H}$, Frontal sections of $\mathrm{Nes}^{\mathrm{Cre} /+} ; R 26^{\mathrm{SmoM2/+}}(\boldsymbol{E}-\boldsymbol{H})$ and $\mathrm{Cre}-$ control $(\boldsymbol{A}-\boldsymbol{D})$ embryos at E12.5. Midline is to the left. Immunofluorescence for Shh shows the location of the ZLI, which is similar between $\mathrm{Nes}^{\mathrm{Cre} /+} ; R 26^{\mathrm{SmoM2}^{\prime++}}(\boldsymbol{A})$ and control brins $(\boldsymbol{E})$. $\boldsymbol{B}, \boldsymbol{F}$, High-magnification views of the boxed areas in $\boldsymbol{A}$ and $\boldsymbol{E}$, respectively. These are adjacent to $\boldsymbol{C} / \boldsymbol{D}$ and $\boldsymbol{G} / \boldsymbol{H}$ respectively. Double immunofluorescence for Nkx2.2 and 0 lig2 $2(\boldsymbol{C}, \boldsymbol{G})$ and double immunofluorescence for Mash 1 and $\mathrm{Ngn2}(\boldsymbol{D}, \boldsymbol{H})$ show that in the control brain, Nkx2.2 and Mash1 are expressed in pTH-R and form a distinct boundary with pTH-C, which expresses 0 lig2 and Ngn2 $(\boldsymbol{C}, \boldsymbol{D})$. In Nes ${ }^{\mathrm{Cr} /+} ; R 26^{\text {SmoM2/++}}$ brains, both Nkx2.2 and Mash1 are expanded caudodorsally, increasing the size of pTH-R. Only in Nes ${ }^{\text {re/+ }} ; R 26^{\text {SmoM2/+ }}$ embryos, there is an additional domain between pTH-R and pTH-C that contain both pTH-Rmarkers Nkx2.2/Mash1 and pTH-C markers Olig2/Ngn2. These two sets of markers are not colocalized (arrows in $\mathbf{G}, \boldsymbol{H}$ ). Dotted lines outline the ZLI in each panel. Arrowheads indicate the domain with both pTH-R and pTH-C markers, which we refer to as PTH-R/C. I, J, Analysis of E12.5 embryos electroporated with PCAG-SmoM2-EYFP plasmid at E11.5 in the thalamus. Double immunofluorescence of Nkx2.2 and YFP on frontal sections. Right side was transfected. Left side is control. Nkx2.2 is ectopically induced in the thalamic progenitor cells only on the transfected side (double arrows in I). Transfection occurred only in the thalamus, not in the prethalamus, as shown by YFP expression $(\boldsymbol{J}) . \boldsymbol{K}$, Strategy for making the 0 lig ${ }^{3{ }^{\text {re }}}$ mice. The exon encoding for Olig3 was replaced by the Cre-frt-neo-frt (Cre-FNF) cassette. L-0, Analysis of recombination in Olig $3^{\text {Cre }}$ mice. Olig ${ }^{3 \text { re/+ }}$ mice were bred with ROSA26-stop-EYFP mice and embryos were analyzed at E11.5. Double immunofluorescence of YFP $(\boldsymbol{L})$ and 0 lig3 $(\boldsymbol{M})$ shows that YFP is expressed in the thalamus, where endogenous Olig3 is expressed. Low-magnification images of the same embryo indicate the specificity of Cre-mediated recombination in the forebrain $(\boldsymbol{N}, \mathbf{O})$. Neither other regions of the diencephalon or the neocortex have undergone recombination (arrows). P-S, Frontal sections of 0 lig $3^{(\mathrm{Cr} /+} ; R 26^{\text {SmoM2/+ }}$ embryos at E12.5, showing that thalamus-specific, ectopic expression of $S \mathrm{moM} 2$ causes the same marker changes in progenitor cells as seen in $\mathrm{Nes}^{\mathrm{Cre} /+} ; R 26^{\mathrm{SmoM} 2 /+}$ embryos $(\boldsymbol{A}-\boldsymbol{H})$. pTH-R is expanded (bracket), and the mixed zone (pTH-R/C) emerges between pTH-R and pTH-C (arrowheads). Scale bar, $200 \mu \mathrm{m}$ for $\boldsymbol{A}-\boldsymbol{M}$ and $\boldsymbol{P}-\boldsymbol{S}, 500 \mu \mathrm{m}$ for $\boldsymbol{N}$ and $\boldsymbol{O}$.

expanded pTH-R (Fig. 3P-S). The results of these two additional approaches, in vivo electroporation and the use of $\mathrm{Olig} 3^{\mathrm{Cre}}$ mice, further reinforce our conclusion that it is the intrinsic increase in Shh signaling that causes the caudodorsal shift of pTH-R markers in thalamic progenitor cells.

We next examined how the pTH-C domain was affected by the increase in Shh signaling. First, in $\mathrm{Nes}^{\mathrm{Cre} /+} ; \mathrm{R}_{2} 6^{\mathrm{SmoM} /+} \mathrm{em}-$ bryos, Olig3 was expanded caudodorsally into the pretectal/ habenular region at E12.5 (Fig. $4 A, G$ ). At this stage, $G b x 2$ and $R O R \alpha$, markers of the postmitotic thalamus derived from the pTH-C domain (Vue et al., 2007), were also expanded accordingly (supplemental Fig. S $3 A-C, F-H$, available at www. 


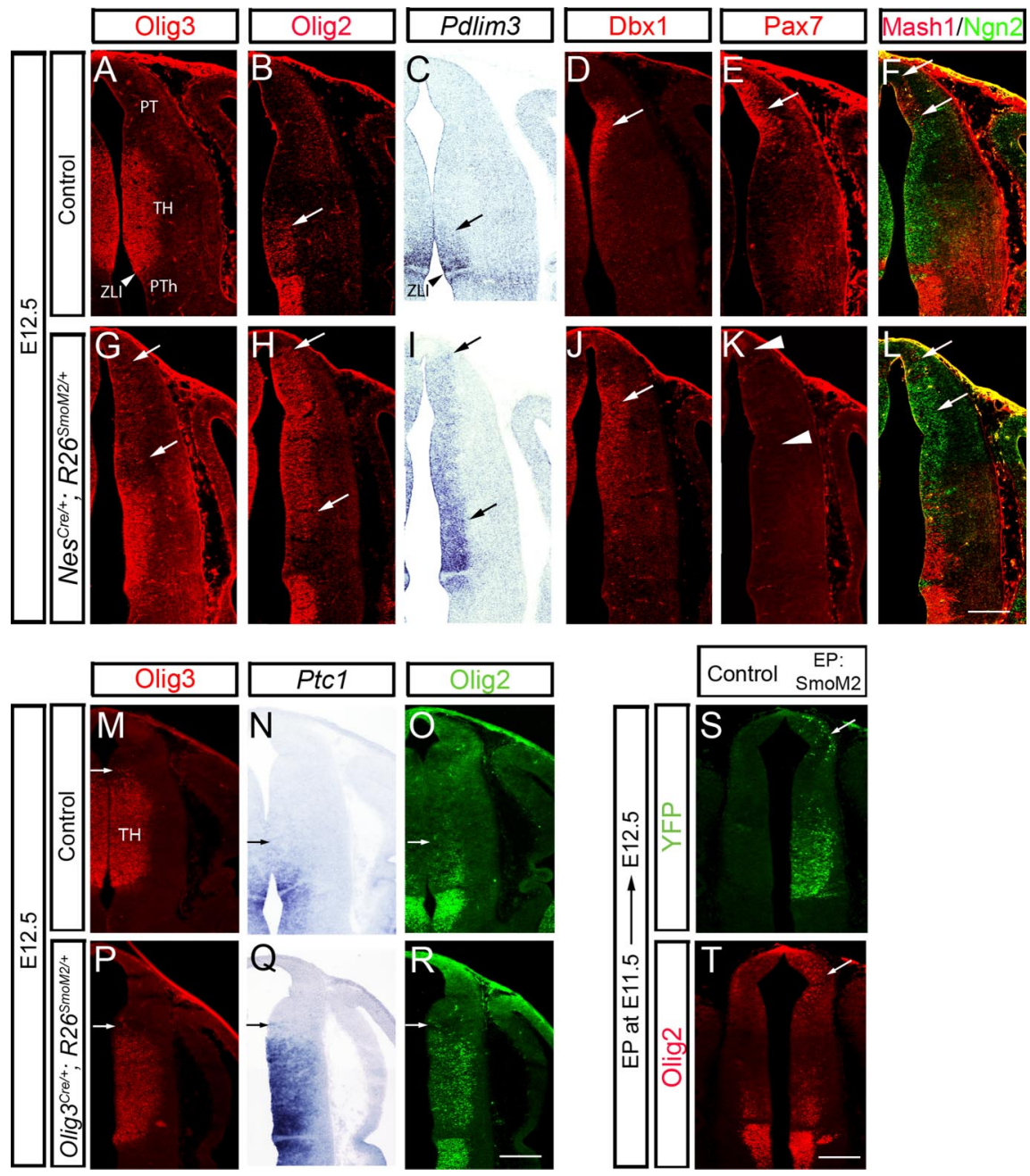

Figure 4. Ectopic SmoM2 expression in neural progenitor cells causes the caudal diencephalon to acquire rostral thalamic identity. $\boldsymbol{A}-\boldsymbol{L}$, Frontal sections of $\mathrm{Nes}^{\mathrm{Cre} /+} ; R 26^{\mathrm{SmoM2/+}}(\boldsymbol{G}-\boldsymbol{L})$ and $\mathrm{Cre}-\operatorname{control}(\boldsymbol{A}-\boldsymbol{F})$ embryos at E12.5. Midline is to the left. In $\mathrm{Nes}^{\mathrm{Cre} /+} ; R 26^{\text {SmoM2/+}}$ thalamus, 0lig3 expression maintains its rostral boundary between the ZLI and the prethalamus, but is expanded caudodorsally ( $\boldsymbol{A}, \mathbf{G}$; arrows in $\mathbf{G}$ indicate the area of expansion). Similarly, 0lig2 and Pdlim3, both of which are

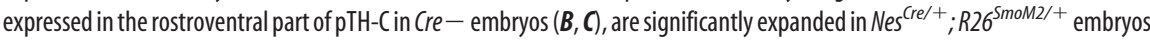
$(\boldsymbol{H}, \boldsymbol{I}$; arrows show the extent of expansion). Dbx 1 , which is normally expressed in the caudal pTH-C and the rostral pretectum ( $\boldsymbol{D}$, arrow), is not significantly altered in $\mathrm{Nes}^{\mathrm{Cre} /+} ; R 26^{\mathrm{SmoM} 2 /+}$ embryos ( $\boldsymbol{J}$, arrows). Pax7 is expressed in the pretectum and the habenula of $\mathrm{Cre}-$ controls $\left(\boldsymbol{E}\right.$, arrow), but is reduced in $\mathrm{Nes}^{\mathrm{Cre} /+} ; R 26^{\mathrm{SmoM} /+}$ embryos ( $\boldsymbol{K}$, no expression between arrowheads). $\mathrm{Ngn} 2$ is expressed in $\mathrm{PTH}-\mathrm{C}$ and the rostral pretectum, whereas Mash1 is expressed in the caudal pretectum ( $\boldsymbol{F}$, arrows to show Mash1 expression). In Nes ${ }^{\text {(re/+ }} ; R 26^{\text {SmoM2/+ }}$ brains, Ngn2 is induced in the caudal pretectum and Mash1 is repressed ( $\boldsymbol{L}$, no expression between the arrows). $\boldsymbol{M}-\boldsymbol{R}, 0$ lig $3^{\text {Cre/ }+} ; R 26^{\text {SmoM2/+ }}(\boldsymbol{P}-\boldsymbol{R})$ and $(r e-\operatorname{control}(\boldsymbol{M}-\mathbf{0})$ embryos at E12.5. Midline is to the left. In Olig ${ }^{\mathrm{Cre} /+} ; R 26^{\text {SmoM2/+ }}$ embryos, Olig3 expression is not expanded, unlike in $\mathrm{Nes}^{\mathrm{Cre} /+} ; R 26^{\text {SmoM2/+}}$ embryos $(\boldsymbol{M}, \boldsymbol{P}$, arrow showing the caudodorsal boundary of $\mathrm{pTH}-\mathrm{C}$ ), indicating that $\mathrm{SmoM} 2$ expression is indeed limited to the thalamus. Within the thalamus, however, Ptc1 expression is increased and now occupies the entire pTH-R and pTH-C $(\mathbf{N}, \mathbf{Q})$. Olig2 is also induced more caudodorsally within pTH-C $(\mathbf{O}, \boldsymbol{R})$. Arrow in $\boldsymbol{M}-\boldsymbol{R}$ indicates the caudal-dorsal limit of gene expression, showing that unlike in the $\mathrm{Cre}$ - control, Ptc 1 and 0 lig2 are now expressed in the entire PTH-C in Olig $3^{\mathrm{Cre} /+} ; R 26^{\text {SmoM2/+ }}$ embryos. S, T, E12.5 embryo in which pCAG-SmoM2-EYFP was electroporated into two focal diencephalic regions at E11.5. The right side was electroporated. 0lig2 is ectopically expressed in the caudal group of electroporated cells in an isolated manner (arrow). Scale bar, $200 \mu \mathrm{m}$.

jneurosci.org as supplemental material). In contrast, Pax7, which is normally expressed in the pretectum and habenula, but not in thalamic progenitor cells, was not detected in $\mathrm{NeS}^{\mathrm{Cre} /+}$; $\mathrm{R}_{2} 6^{\mathrm{SmoM} 2 /+}$ embryos (Fig. 4 E, K). Moreover, expression of Mash1 in the caudal pretectum was replaced by Ngn2, which normally spans from pTH-C only into the rostral pretectum (Fig. 4F,L). Irx2 and BHLHB4, early postmitotic markers of the pretectum, were also reduced at E12.5 (supplemental Fig. S3 D, E, I, J, available at www.jneurosci.org as supplemental material). These results show that a high Shh signal in the caudal diencephalon induces the thalamic progenitor domain, pTH-C, at the expense of the pretectum and habenula. Olig2, which is normally expressed in a rostral-ventral to caudal-dorsal gradient within $\mathrm{pTH}-\mathrm{C}$, was homogeneously expressed in this expanded pTH-C domain of $\mathrm{Nes}^{\mathrm{Cre} /+} ; \mathrm{R}_{2} 6^{\mathrm{SmoM} 2 /+}$ embryos (Fig. $4 B, H$ ). Another marker gene, Pdlim3, which is normally expressed in pTH-R as well as the rostroventral part of pTH-C (Fig. 4C) (Gray et al., 2004), also expanded (Fig. 4C,I). These results demonstrate that enhanced Shh signaling in the entire diencephalon transformed it into the rostral-ventral thalamus, which is composed of the pTH-R domain and the rostral-ventral part of the pTH-C domain (summarized in Fig. $9 A, B 1, B 2, C 1, C 2)$.

Surprisingly, the expression of Dbx1, which is normally expressed in the caudodorsal part of pTH-C as well as in the pretectum and habenula, was not significantly reduced in $\mathrm{NeS}^{\mathrm{Cre} /+} ; \mathrm{R}_{2} 6^{\mathrm{SmoM} 2 /+}$ embryos (Fig. 4D, J), suggesting that Dbx1 is positively regulated by some unknown mechanisms operating in the caudodorsal diencephalon. Expression of Dlx2, a prethalamic marker, was not ectopically induced in the thalamic progenitor cells in $\mathrm{Nes}^{\mathrm{Cre} /+}$; $\mathrm{R}_{2} 6^{\mathrm{SmoM} 2 /+}$ embryos (data not shown), consistent with a previous study showing that the thalamus and the prethalamus are distinctly prepatterned and express different sets of genes in response to Shh signaling (Kiecker and Lumsden, 2004).

To ensure that the above changes were also not the product of indirect effects, we again analyzed embryos electroporated with the $p C A G-S m o M 2-$ EYFP plasmid, as well as $\mathrm{Olig}^{\mathrm{Cre} /+}$; $R 26^{\text {SmoM2/+ }}$ embryos. In electroporated embryos where an isolated population of progenitor cells in the pretectum were transfected, Olig2 was ectopically induced (Fig. 4S,T). In $\mathrm{Olig}^{\mathrm{Cre} /+}$; $R 26^{\text {SmoM2/+ }}$ embryos, although the pretectum and the habenula are spared and the extent of Olig3 expression was similar to controls (Fig. 4M,P), Ptcl and Olig2 were homogeneously elevated within the entire pTH-C domain (Fig. $4 N, O, Q, R)$, demonstrating that thalamus-specific elevation of Shh signaling intrinsically induced the rostral pTH-C markers.

\section{Expression of rostral postmitotic thalamic markers is expanded in SmoM2-expressing embryos}

Does increased Shh signaling in thalamic progenitor cells lead to reorganization of postmitotic thalamus? To address this question, we examined the expression of postmitotic markers that are 
differentially expressed within nuclei of the late embryonic thalamus (Fig. 5).

First, we analyzed nuclei that are derived from the pTH-R domain. We previously found that progenitor cells in pTH-R generate part of the vLG as well as the IGL, two nuclei that do not contain neurons projecting to the neocortex (Vue et al., 2007). Consistent with this fate analysis and the expanded pTH-R markers in $\mathrm{Nes}^{\mathrm{Cre} /+}$; $\mathrm{R}_{2} 6^{\mathrm{SmoM} 2 /+}$ as well as $\mathrm{Olig} 3^{\mathrm{Cre} /+}$; R26 $6^{\text {SmoM2/+ }}$ embryos, vLG/IGL was expanded caudodorsally along the lateral surface of the diencephalon (Fig. 5A, G for $\mathrm{Nes}{ }^{\mathrm{Cre} /+} ; \mathrm{R}_{2} 6^{\mathrm{SmoM} 2 /+}$, and data not shown for $\mathrm{Olig} 3^{\mathrm{Cre} /+}$; $226^{\mathrm{SmoM} 2 /+}$ ). These expanded nuclei are marked by expression of Nkx2.2, Sox14 (Fig. 5A-C,G-I), and Sox1 (data not shown). The expanded Nkx2.2 and Sox14 expression is consistent with previous studies using chick and mouse embryos (Hashimoto-Torii et al., 2003; Kiecker and Lumsden, 2004; Vieira et al., 2005) and establishes that IGL and the lateral part of vLG are specified by high Shh activity in pTH-R. Nkx2.2-positive cells were also found in a more caudolateral region that is away from the large cluster of expanded vLG/IGL cells (Fig. $5 H$, arrowheads). These cells did not express Sox2 but intermingled with Sox2-expressing cells (Fig. 5, compare $G, H, J$ ). This intermingling of two molecularly different cell types reflects our earlier observation that two completely separate progenitor cell populations, one with pTH-R identity and the other with pTH-C identity, are mixed in the SmoM2-expressing, thalamic ventricular zone (Fig. $3 G, H$ ).

Next, we tested whether markers of thalamic nuclei that are derived from the pTH-C domain are also shifted in SmoM2-expressing embryos. In control embryos, Sox 2 is expressed in the rostroventral part of the pTH-C-derived thalamic mantle zone (Fig. 5D, M,O) (Vue et al., 2007). In E16.5 Nes ${ }^{\mathrm{Cre} /+} ; \mathrm{R}_{2} 6^{\mathrm{SmoM} 2 /+}$ brains, Sox 2 expression was expanded caudally and dorsally, occupying essentially the entire diencephalic mantle zone (Fig. $5 D, J$ ). Similarly, another rostral nucleus marker, $R O R \alpha$, which is expressed more specifically in ventral posterior nucleus (VP) and dLG (Nakagawa and O'Leary D, 2003), was expanded caudally and dorsally in $\mathrm{NeS}^{\mathrm{Cre} /+} ; \mathrm{R}^{\mathrm{SmoM} 2 /+}$ brains compared with controls (Fig. $5 E, K$ ). To determine whether the expansion of these rostral nucleus markers is accompanied by the repression of caudal nucleus markers, we analyzed the expression of Gbx2. Although $G b \times 2$ is initially expressed in the entire postmitotic thalamus (supplemental Fig. $\mathrm{S} 3 A, B, F, G$, available at www. jneurosci.org as supplemental material), its expression later becomes restricted to medial and caudodorsal nuclei and is nearly
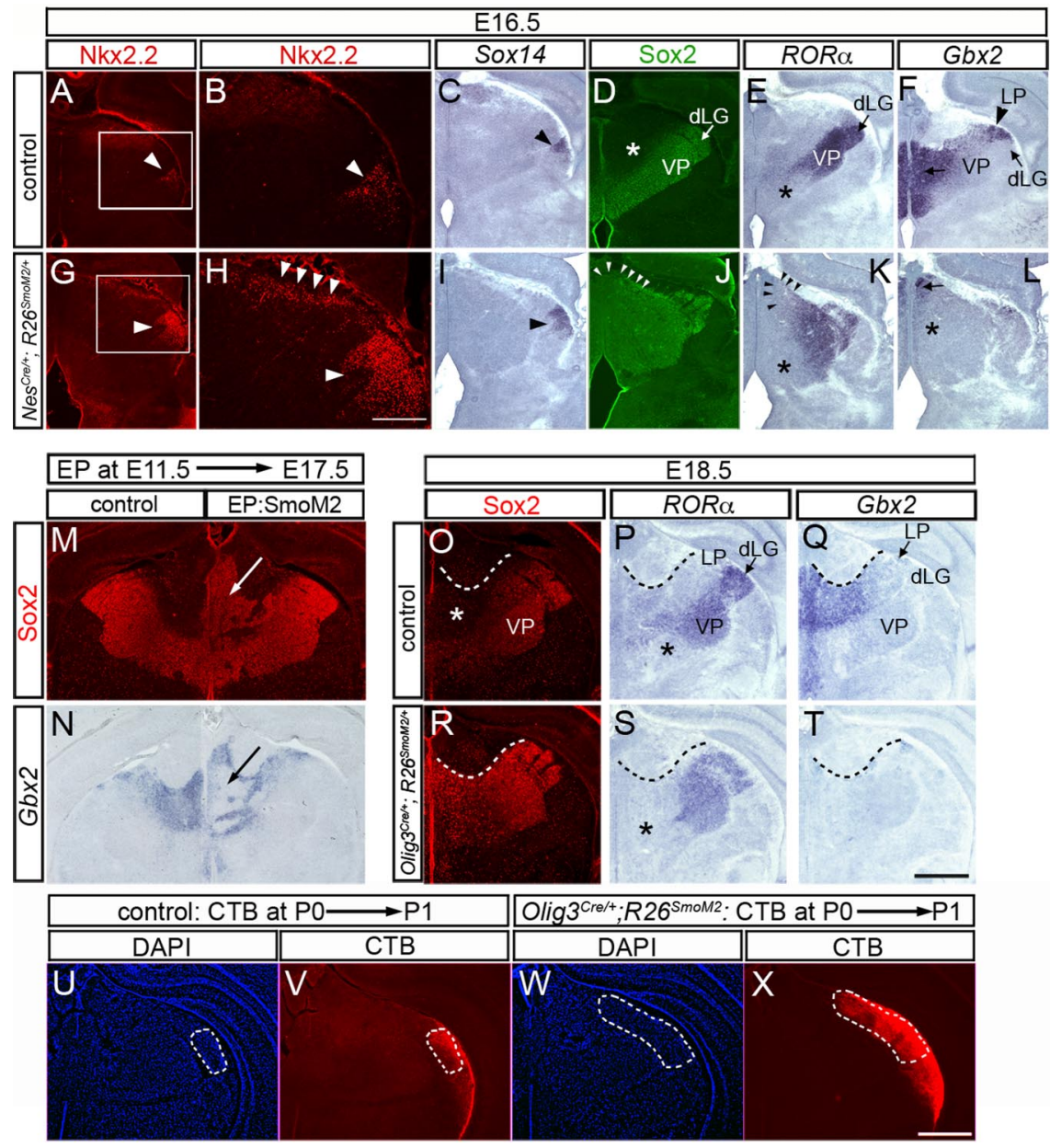

Figure 5. Ectopic SmoM2 expression causes a rostroventral shift of postmitotic thalamus. $A-L$, Frontal sections of $\mathrm{Nes}^{\mathrm{Cre} /+}$; $R 26^{\text {SmoM2/+ }}(\boldsymbol{G}-\boldsymbol{L})$ and $(r e-$ control embryos $(\boldsymbol{A}-\boldsymbol{F})$ at E16.5. Midline is to the left. In control embryos, Nkx2.2 and Sox14 expression delineates the lateral VLG and IGL $(\boldsymbol{A}-\boldsymbol{C}$, arrowhead), which are derived from pTH-R. $\boldsymbol{B}$, High-magnification image of the boxed region in $\boldsymbol{A}$. In Nes ${ }^{\mathrm{Cre} /+} ; R 26^{\text {SmoM2/+ }}$ embryos, Nkx2.2 and Sox14 expression is expanded caudally and dorsally (G-I, arrowhead). $\boldsymbol{H}$, High-magnification image of the boxed areas in $\boldsymbol{G}$, showing that some cells ectopically expressing Nkx2.2 ( $\boldsymbol{H}$, arrowheads) are intermingled with Sox2-expressing cells $(\boldsymbol{J})$. In (re-controls, Sox 2 is highly expressed in rostroventral part of the postmitotic thalamus, including VP and dLG, but not in more caudodorsal part ( $\boldsymbol{D}$, asterisk). In $\mathrm{Nes}^{\mathrm{Cre} /+} ; \mathrm{R2}^{\mathrm{SmoM2/+}} \mathrm{embryos}^{\mathrm{B}}$ Sox2 is robustly expanded caudodorsally ( $\boldsymbol{J}$, arrowheads). Similarly, $R O R \alpha$ expression, which corresponds to rostrolateral nuclei such as VP and dLG $(\boldsymbol{E})$, is also expanded caudodorsally in $\mathrm{Nes}^{\mathrm{Cre} /+} ; R 26^{\mathrm{SmoM2} /+}$ embryos ( $\boldsymbol{K}$, arrowheads). Note that the medial part of the thalamus that is negative for $R O R \alpha$ in control is also negative in $\mathrm{SmoM} 2$ embryos $(\boldsymbol{E}, \boldsymbol{K}$, asterisk). The caudodorsal expression border of $G b x 2$ is elongated near the midline in $5 m 0 M 2$ embryos $(\boldsymbol{F}, \boldsymbol{L}$, arrow), but overall, Gbx2 expression is reduced, especially from the medial dorsal part $(\boldsymbol{F}, \boldsymbol{L}$, asterisk). $\boldsymbol{M}, \boldsymbol{N}, \mathrm{E} 17.5$ embryo in which $p$ CAG-SmoM2-EYFP plasmid was electroporated at E11.5 into the thalamus. The right side was electroporated, and the left side is the control. On the electroporated side, Sox2 is ectopically induced in a caudodorsal region $(\boldsymbol{M}$, arrow), while this induction did not occur on the control side. Conversely, Gbx2 is downregulated where Sox2 is induced ( $\boldsymbol{N}$, arrow). $\mathbf{0}-\boldsymbol{T}, 0 \mathrm{Olig} 3^{\mathrm{Cre} /+} ; R 26^{\mathrm{SmoM} 2 /+}$ and $\mathrm{Cre}-$ control embryos at E18.5. Midline is to the left. In Olig $3^{\mathrm{Cre} /+} ; R 26^{\text {SmoM2/+ }}$ embryos, Sox2 expression is expanded and now occupies the entire thalamic mantle zone $(\boldsymbol{R})$. The Sox2-negative, caudodorsal region (asterisk in $\mathbf{0}$ ) is undetectable in 0 lig $3^{\mathrm{Cre} /+} ; R 26^{\mathrm{SmoM2/+}}$ embryos. The caudodorsal boundary of the thalamus is outlined by a dotted line. Similarly, expression of $R O R \alpha$ is expanded $(\boldsymbol{P}, \mathbf{S})$. Unlike Sox2, ROR $\alpha$ is not induced in the medial part of the thalamic mantle zone $(\boldsymbol{P}, \mathbf{S}$, asterisk). Gbx2 expression is dramatically reduced in 0 lig $3^{\mathrm{Cre} /+} ; R 26^{\text {SmoM2/+ }}$ embryos $(\boldsymbol{Q}, \boldsymbol{T}) . \boldsymbol{U}-\boldsymbol{X}$, Postnatal day 1 thalamus of 0 lig $3^{\mathrm{Cre} /+} ; R 26^{\mathrm{SmoM} 2 /+}$ $(\boldsymbol{W}, \boldsymbol{X})$ and $C$ re - control $(\boldsymbol{U}, \boldsymbol{V})$ pups injected with CTB in the contralateral retina at PO. dLG nucleus is delineated as dashed lines by CTB immunohistochemistry $(\boldsymbol{V}, \boldsymbol{X})$ and DAPI staining $(\boldsymbol{U}, \boldsymbol{W}) . \mathrm{dLG}$ is expanded by $\sim 3.6$ times (see Results). Scale bar, $200 \mu \mathrm{m}$ for $\boldsymbol{B}, \boldsymbol{H} ; 500 \mu \mathrm{m}$ for other panels.

complementary to that of Sox 2 and $R O R \alpha$ (Fig. $5 F, N, Q)$. In $\mathrm{NeS}^{\mathrm{Cre} /+}$; R26 $6^{\mathrm{SmoM} 2 /+}$ brains, Gbx2 expression was dramatically reduced, particularly in regions where both Sox 2 and $R O R \alpha$ are ectopically induced (Fig. 5L).

To better demonstrate the local effects of enhanced Shh signal on postmitotic thalamic development, we again analyzed SmoM2-electroporated embryos and $\mathrm{Olig} 3^{\mathrm{Cre} /+} ; \mathrm{R}_{2} 6^{\mathrm{SmoM} 2 /+} \mathrm{em}-$ bryos. When caudodorsal thalamus was electroporated at E11.5 
and analyzed at E17.5, Sox2 was highly induced in the cohort of cells on the transfected side (Fig. 5M). Gbx2 was specifically repressed in these Sox2-expressing cells, but its expression on the control side was unaffected (Fig. $5 \mathrm{M}, \mathrm{N}$ ). In $\mathrm{Olig}^{\mathrm{Cre} /+}$; $R 26^{\text {SmoM2/+ }}$ brains at E18.5, the overall morphology of the thalamic mantle zone was normal. However, Sox2 was ubiquitously expressed in the entire thalamus, not just in rostroventral nuclei as seen in the control (Fig. 5O,R). $R O R \alpha$ was also significantly expanded caudodorsally in the lateral part of the thalamus (Fig. $5 P, S)$. Furthermore, $G b \times 2$ expression is almost completely absent in $\mathrm{Olig} 3^{\mathrm{Cre} /+} ; \mathrm{R}^{\mathrm{SmoM} 2 /+}$ brains (Fig. 5Q,T). These data demonstrate that in $\mathrm{Olig} 3^{\mathrm{Cre} /+} ; \mathrm{R}_{2} 6^{\mathrm{SmoM} 2 /+}$ embryos, the entire postmitotic thalamus is dominated by rostroventral identity.

Although the expansion of $R O R \alpha$ implicates that rostroventrally located nuclei, VP, and $\mathrm{dLG}$ are increased in size in Olig $3^{\mathrm{Cre} /+} ; \mathrm{R}_{2} 6^{\mathrm{SmoM} 2 /+}$ embryos, we used criteria other than molecular markers that would definitively prove that individual nuclei are altered. For this purpose, we used anatomical criteria to define thalamic nuclei. Specifically, we injected anterograde axon tracer CTB to the retina of the neonatal Olig $3^{\mathrm{Cre} /+} ; \mathrm{R}_{2} 6^{\mathrm{SmoM} 2 /+}$ mice to label retinogeniculate axons in the $\mathrm{dLG}$ nucleus. Because Olig3 is not expressed in the retina or along the retinogeniculate pathway to the thalamus, $\mathrm{Olig} 3^{\mathrm{Cre} /+} ; \mathrm{R}^{\mathrm{SmoM} 2 /+}$ mice provide a particularly useful model to evaluate the specific effects of enhanced Shh signal in thalamic progenitor cells on the size of postnatal thalamic nuclei. We found that the volume of the dLG nucleus as estimated by the CTB-labeled region in the thalamus, was increased by $\sim 3.6$ times in Cre+ pups $\left(0.086 \pm 0.008 \mathrm{~mm}^{3}\right.$ in Cre + pups, $n=3 ; 0.024 \pm 0.003 \mathrm{~mm}^{3}$ in Cre-pups, $n=2$; $p=0.0091)($ Fig. $5 U-X)$.

In summary, we demonstrated that increasing Shh signaling in CNS progenitor cells results in expansion of rostral-ventral thalamic progenitor pools, $\mathrm{pTH}-\mathrm{R}$, and rostroventral $\mathrm{pTH}-\mathrm{C}$, at the expense of more caudal-dorsal thalamus as well as the pretectum and habenula (summarized in Fig. 9B1,B2,C1,C2). When the manipulation of the Shh signal was restricted to thalamic progenitor cells, pTH-R and rostroventral pTH-C domain still expanded and caudal-dorsal pTH-C was shrunken. These results strongly indicate that the high Shh signal biases thalamic progenitor cells toward more rostral-ventral fates in a cell-intrinsic manner. This conclusion is consistent with our observation that the number of $\mathrm{PH} 3+$ cells in the entire Olig3-expressing domain was similar between $\mathrm{Olig}^{\mathrm{Cre} /+} ; \mathrm{R}^{\mathrm{SmoM} 2 /+}$ and control embryos (supplemental Fig. S2, available at www.jneurosci.org as supplemental material). Compatible with changes in progenitor identity, postmitotic thalamic nuclei are expanded or shrunken based on their putative locations of origin (summarized in Fig. 9B3,C3).

Because Gli1 and Ptc1 are not ectopically expressed in the mantle zone of SmoM2-expressing brains, the altered organization of the postmitotic thalamus is unlikely to be due to the direct activation of Shh signaling in postmitotic cells. Instead, we conclude that shifted identity of thalamic progenitor cells resulted in corresponding alteration of the identity of postmitotic thalamic nuclei.

\section{Overexpression of the bHLH transcription factor Olig2 partially mimics the effect of SmoM2 expression in the thalamus}

If Shh signal directly controls the identity of thalamic progenitor cells and indirectly influences the specification of postmitotic thalamic nuclei, it is likely that transcription factors that are regulated by Shh signal in progenitor cells translate their positional information onto their postmitotic progeny. Olig2 is normally expressed in the rostroventral portion of pTH-C, and is induced in progenitor cells that ectopically express SmoM2. These SmoM2-expressing embryos also showed induction of Sox 2 and $R O R \alpha$ caudally and dorsally throughout the thalamic mantle zone. Thus, it is possible that Olig2 functions to specify rostroventrally located thalamic nuclei that express Sox 2 and $R O R \alpha$. To test this possibility, we first performed genetic lineage tracing of Olig2-expressing progenitor cells (supplemental Fig. S4A-C, available at www.jneurosci.org as supplemental material). We crossed Olig2 ${ }^{\mathrm{Cre} /+}$ mice (Dessaud et al., 2007) with ROSA26-stopEGFP reporter mice, and analyzed E14.5 compound heterozygous embryos. At E11.5, GFP was expressed in the rostroventral part of pTH-C, as well as pTH-R (supplemental Fig. S4A, available at www.jneurosci.org as supplemental material). Expression of GFP in pTH-R suggests that there is transient expression of Olig2 in this progenitor domain before E10.5, which is similar to the observation in ventral spinal cord (Dessaud et al., 2007). At E14.5, EGFP was expressed in the rostroventral portion of the postmitotic thalamus, which largely overlapped with Sox2 expression (supplemental Fig. S4 B, C, available at www.jneurosci. org as supplemental material). VP and the ventral part of medial geniculate $(\mathrm{MGv})$ nuclei were two nuclei that had particularly strong EGFP expression. In contrast, EGFP was not found in more caudodorsal nuclei. Thus, Olig2-expressing thalamic progenitor cells give rise to rostroventral part of the postmitotic thalamus, consistent with the possibility that Olig2 plays a role in the specification of rostroventral nuclei such as VP and MGv.

We then tested whether Olig2 is necessary and/or sufficient for the specification of these nuclei. When we ectopically expressed Olig2 by in utero electroporation at E10.5 in the caudodorsal diencephalon, Sox2 was induced in the mantle zone only on the transfected side at E13.5 and E14.5 (supplemental Fig. $\mathrm{S} 4 E, F$, available at www.jneurosci.org as supplemental material), which partially mimicked the effect of expressing SmoM2. Cell proliferation and neuronal differentiation did not seem to be affected in Olig2-transfected cells, and overexpressing EGFP alone did not induce Sox2 expression (data not shown). These results suggest that Olig2 mediates the Shh signal and confers at least part of the rostroventral identity to the postmitotic thalamus. In contrast, Olig2 null mice did not show altered expression of Sox2 or progenitor markers such as Mash1, Ngn2, and Dbx1 (data not shown). Thus, Olig2 is not necessary but is sufficient to induce at least some molecular features of the rostroventral thalamic nuclei.

\section{Attenuation of Shh signal in thalamic progenitor cells reduces the expression of rostroventral markers and increases caudodorsal markers}

Previous studies on chick embryos showed that ectopic expression of Ptc1 $\Delta$ loop2, a dominant-negative form of the Shh receptor, Ptc1, reduced the expression of $N k \times 2.2$ and Gbx2, and increased the expression of Pax6, which is normally expressed highly in the caudal diencephalon (Kiecker and Lumsden, 2004). It was also reported that Gli2/3 double knock-out mice showed reduced expression of $G b \times 2$, whereas Gli1 mutant mice showed reduced Sox14 expression (Hashimoto-Torii et al., 2003). These studies demonstrated the requirement of Shh signaling in establishing the identity of the thalamus as a whole. However, it is still unknown whether Shh is required for the patterned expression of progenitor markers in $\mathrm{pTH}-\mathrm{R}$ and $\mathrm{pTH}-\mathrm{C}$, or for the normal organization of postmitotic thalamic nuclei. Shh null mice show pronounced reduction in size of the diencephalon by E9.0, which reflects the early role of Shh in cell proliferation and survival 
(Ishibashi and McMahon, 2002). Therefore, we analyzed conditional Shh knockout mice by using the Nes ${ }^{\text {Cre }}$ allele to avoid such early effects. Conditional Shh mutant mice were smaller than control littermates, but they were postnatally viable and have relatively normal gross morphology of the brain (data not shown). The smaller brain size is reflected in part by a significant decrease in the number of $\mathrm{PH} 3$-positive progenitor cells in the thalamic ventricular zone of conditional Shh knock-out $\left(\mathrm{Nes}^{\mathrm{Cre} /+} ; \mathrm{Shh}^{c / c}\right)$ embryos (3437 \pm 146.0 , $n=2$ for $\mathrm{Nes}^{\mathrm{Cre} /+}$; Shh ${ }^{c / c}, 6605 \pm 472.0$, $n=2$ for Cre- controls; $p=0.024$ ) (supplemental Fig. S2C,D, available at www. jneurosci.org as supplemental material).

In $\mathrm{Nes}^{\mathrm{Cre} /+}$; $\mathrm{Shh}^{c / c}$ embryos, Shh expression in the forebrain was lost by E10.5 (data not shown; Machold et al., 2003). Consistent with the loss of Shh in the ZLI and the basal plate, Ptcl (data not shown) and Gli1 mRNA was undetectable at E10.5 and E12.5 in Nes ${ }^{\mathrm{Cre} /+}$; $\mathrm{Shh}^{c / c}$ embryos (Fig. $6 B, G$ and not shown). Furthermore, markers for the pTH-R domain were not induced. At E12.5, we observed a complete loss of Nkx2.2 (Fig. 6C,H) and Mash1 (Fig. $6 D, I)$ within the thalamic ventricular zone, although their expression in the prethalamic progenitor domain remained at very low levels (Fig. 6H,I). Thus, $\mathrm{Nes}{ }^{\mathrm{Cre} /+}$ mice are useful in deleting the Shh gene in the brain when Shh signal is still critically required for the induction and/or maintenance of the expression of pTH-R markers. In addition to Nkx2.2 and Mash1, expression of Olig2, a rostroventral pTH-C marker, was also lost in pTH-C of Nes ${ }^{\mathrm{Cre} /+} ; \mathrm{Shh}^{c / c}$ embryos, although it was weakly detected in the prethalamus (Fig. 6C,H, arrowhead for the prethalamus). In contrast, markers that are expressed throughout the $\mathrm{pTH}-\mathrm{C}$ domain, such as Olig3 (Fig. 6A,F) and Ngn2 (Fig. 6D,I) were still expressed in $\mathrm{Nes}^{\mathrm{Cre} /+}$; $\mathrm{Shh}^{c / c}$ embryos at E12.5 and earlier stages, although the size and intensity of the expression of Olig3 seemed to be reduced. Dbx1, which is normally restricted to the caudodorsal pTH-C, was expanded rostroventrally in $\mathrm{Nes}^{\mathrm{Cre} /+}$; $S h h^{c / c}$ thalamus (Fig. 6E,J). A pretectal marker, Pax7, was still expressed (data not shown). Together, these findings demonstrate that Shh activity is required for progenitor cell identity in pTH-R and rostroventral pTH-C and for suppressing the caudodorsal pTH-C identity (summarized in Fig. 9B1,B2,D1,D2).

Next, to more directly test the intrinsic requirement of Shh signaling in the thalamus, we conditionally knocked out the gene encoding Smo, a transmembrane effector of Shh signaling. Two Cre drivers, $\mathrm{Nes}^{\mathrm{Cre} /+}$ and $\mathrm{Olig} 3^{\mathrm{Cre} /+}$, were used. Both $\mathrm{NeS}^{\mathrm{Cre} /+}$; $\mathrm{Smo}^{c / c}$ and $\mathrm{Olig}^{\mathrm{Cre} /+}$; $\mathrm{Smo}^{c / c}$ mice showed normal gross morphology of the brain and survived postnatally. We found that $\mathrm{NeS}^{\mathrm{Cre} /+}$; $\mathrm{Smo}^{c / c}$ embryos show lack of Ptcl expression in the thalamus at E12.5 (Fig. $7 B, G$ ) despite the normal expression of Shh in the ZLI (Fig. $7 A, F$ ). In these embryos, we also found that Olig3 expression was reduced in the thalamic ventricular zone (Fig. $7 A, F)$, and Mash1 and Olig2 were undetectable in pTH-R and pTH-C, respectively (Fig. 7C,D,H,I). Furthermore, Dbx1 expression was expanded rostroventrally (Fig. 7 E,J). However, un-
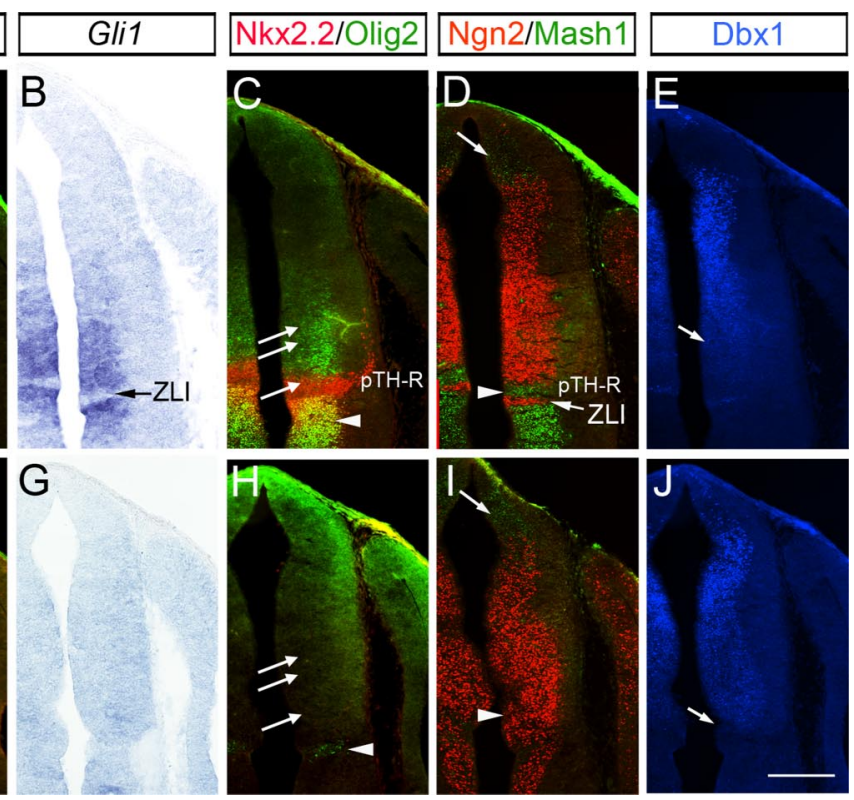

Figure 6. Shh is required for the normal organization of thalamic progenitor domains. Frontal sections of $\mathrm{Nes}^{\mathrm{Cre} /+} ; \mathrm{Shh}^{\mathrm{c} / \mathrm{C}}$ lost in the thalamic progenitor cells of $\mathrm{Nes}^{\mathrm{Cre} /+} ; \operatorname{Shh}^{\mathrm{c} / \mathrm{C}}$ embryos $(\boldsymbol{G})$, while it is expressed in a graded manner in the control $(\boldsymbol{B})$.

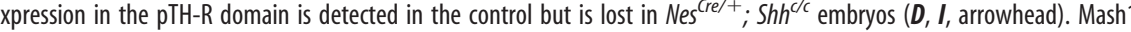
dodorsal part of pTH-C in control embryos $(\boldsymbol{E})$. The expression domain of Dbx1 is expanded rostroventrally in $\mathrm{Nes}^{\mathrm{Cre} /+} ; \mathrm{Shh}^{\mathrm{c} / \mathrm{C}}$ embryos ( $\boldsymbol{E}, \boldsymbol{J}$, arrow). Scale bar, $200 \mu \mathrm{m}$.

like in $\mathrm{NeS}^{\mathrm{Cre} /+}$; $\mathrm{Shh}^{c / c}$ embryos, Nkx2.2 was still detectable though not as robustly as in the control (Fig. 7C,H).

Olig $3^{\mathrm{Cre} /+} ; \mathrm{Smo}^{c / c}$ embryos at E12.5 showed normal expression of Shh in the ZLI (Fig. $7 K, P$ ) and reduction of Ptc1 expression in the thalamus but not in the prethalamus (Fig. $7 L, Q$ ), compatible with the restricted deletion of Smo and reduced Shh signal specific to the thalamus. The reduction of $P t c 1$ expression in the thalamus was less significant than in $\mathrm{Nes}^{\mathrm{Cre} /+} ; \mathrm{Smo}^{c / c} \mathrm{em}-$ bryos, where Ptc1 was undetectable in the thalamus and the prethalamus (Fig. 7G). Nevertheless, Olig ${ }^{\mathrm{Cre} /+} ; \mathrm{Smo}^{c / c}$ embryos showed reduction of Mash1-expressing cells in the thalamus (Fig. $7 M, N, R, S)$. In addition, the decreased number of Mash1expressing cells were intermingled with Ngn2-expressing cells, giving rise to the mixed zone ( $\mathrm{pTH}-\mathrm{R} / \mathrm{C}$ ) instead of pure $\mathrm{pTH}-\mathrm{R}$ as we found in the control (Fig. 7N,S). Although Nkx2.2 and Olig2 were not consistently reduced in expression, Dbx1 expression was grossly expanded rostroventrally (Fig. 7O,T), as in the $\mathrm{Nes}^{\mathrm{Cre} /+}$; $\mathrm{Shh}^{c / \mathrm{c}}$ and $\mathrm{Nes}^{\mathrm{Cre} /+}$; $\mathrm{Smo}^{c / c}$ embryos. Together, these results indicate that the Shh signal is intrinsically required for the induction and/or maintenance of molecular characteristics of the pTH-R domain and for the suppression of caudodorsal part of the pTH-C domain.

The dramatic changes in the progenitor cell identity with conditional deletion of the Shh or Smo gene prompted us to examine which populations of postmitotic thalamus are affected in the absence of Shh or Smo. In E17.5 Nes ${ }^{\mathrm{Cre} /+}$; Shh ${ }^{c / c}$ embryos, as expected from the loss of pTH-R markers at E12.5 (Fig. $6 C, D, H, I), \mathrm{Nkx} 2.2$ (Fig. $8 A, C, F, H$ ) and Sox14 (data not shown) in $v L G / I G L$ were both dramatically reduced, confirming the re- 

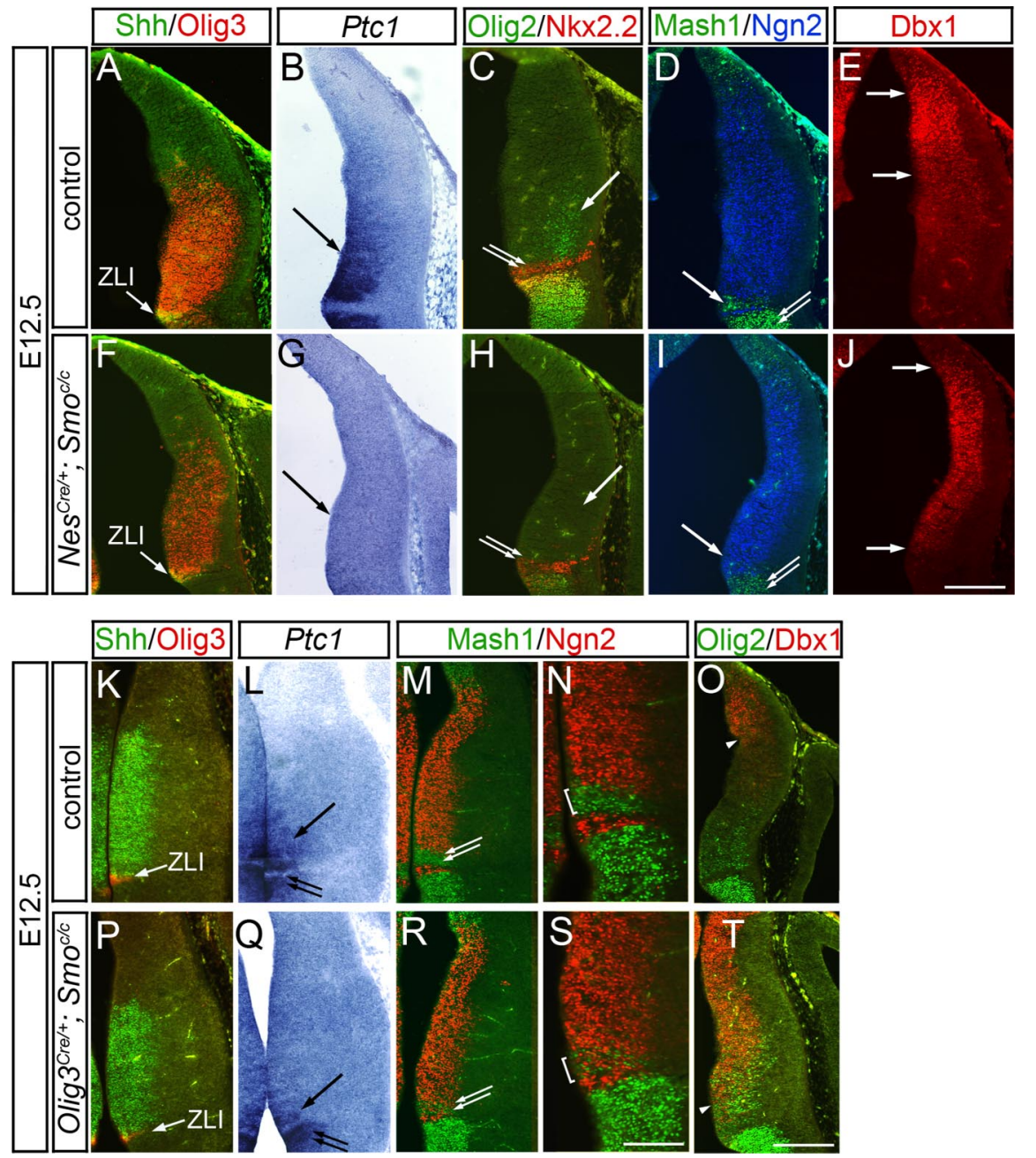

Figure 7. Smo is required for the normal organization of thalamic progenitor domains. Frontal sections of $\mathrm{Nes}^{\mathrm{cre} /+} ; \mathrm{Smo}^{c / c}$ and $\mathrm{Nes}^{\mathrm{Cre} /+} ; \mathrm{Smo}^{\mathrm{C/}+}$ control embryos $(\boldsymbol{A}-\boldsymbol{J})$ and $\mathrm{Olig} 3^{\mathrm{Cre} /+} ; \mathrm{Smo}^{\mathrm{c/c}}$ and $\mathrm{Olig} 3^{\mathrm{Cre} /+} ; \mathrm{Smo}^{\mathrm{c/+}}$ control embryos $(\boldsymbol{K}-\boldsymbol{T})$ at E12.5. Midline is to the left. $\boldsymbol{A}-\boldsymbol{J}, \mathrm{Nes}^{\mathrm{Cre} /+} ; \mathrm{Smo}^{c / c}$ embryos show normal expression of 0 lig3 3 and $\operatorname{Shh}(\boldsymbol{A}, \boldsymbol{F})$, but $\operatorname{Ptc} 1(\boldsymbol{B}, \boldsymbol{G}$, arrow) and 0 lig2 (C, $\boldsymbol{H}$, arrow) are lost in thalamic progenitor cells. Nkx2.2 is still detectable in a reduced number ( $\boldsymbol{C}, \boldsymbol{H}$ double arrows). Mash1 expression in $\mathrm{pTH}-\mathrm{R}$ is undetectable in $\mathrm{Nes}^{\mathrm{Cre} /+} ; \mathrm{Smo}^{\mathrm{c/c}}$ embryo $(\boldsymbol{D}, \boldsymbol{I}$, arrow), whereas it is still detectable in the prethalamus $(\boldsymbol{D}$, $\boldsymbol{I}$, double arrows). Dbx1 expression is expanded in $\mathrm{Nes}^{\mathrm{Cre} /+} ; \operatorname{Smo}^{\mathrm{c} / \mathrm{C}}$ embryos $(\boldsymbol{E}, \boldsymbol{J})$. Arrows in $\boldsymbol{E}$ and $\boldsymbol{J}$ indicate the boundaries of the expression domain of Dbx1. $\boldsymbol{K}-\boldsymbol{T}, \mathrm{Olig}^{\text {(re/ }+} ;$; Smo ${ }^{c / c}$ embryos show normal expression of 0 lig3 and Shh $(\boldsymbol{K}, \boldsymbol{P})$. Ptc 1 expression is reduced in the thalamus $(\boldsymbol{L}, \mathbf{Q}$, arrow) but not in the prethalamus $(\boldsymbol{L}, \mathbf{Q}$, double arrows). Mash1 expression in the thalamus is reduced in $\mathrm{Olig}^{\text {(re/ }}{ }^{+}$; Smo ${ }^{c / c}$ embryos ( $\boldsymbol{M}, \boldsymbol{R}$, double arrow). In high-magnification views of $\boldsymbol{M}$ and $\boldsymbol{R}, \mathrm{Ngn} 2$, a pTH-C marker, is now expressed more rostroventrally. Ngn2-expressing cells are intermingled with Mash1-expressing cells, forming a mixed zone of pTH-R/C at the expense of pTH-R (N, S, bracket). Expression of Dbx 1 is expanded in 0 lig $3^{\text {cre/ }+} ; S_{m o} 0^{c / c}$ embryos, reaching near

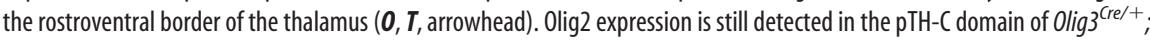
Smo ${ }^{c / c}$ embryos $(\mathbf{O}, \boldsymbol{T})$. Scale bar, $100 \mu \mathrm{m}$ for $\boldsymbol{N}, \mathbf{S} ; 200 \mu \mathrm{m}$ for other panels.

quirement of Shh signaling for the vLG/IGL lineage. Similar changes were found in $\mathrm{Nes}^{\mathrm{Cre} /+}$; $\mathrm{Smo}^{c / c}$ embryos (Fig. $8 \mathrm{~K}-\mathrm{N}$ ). We next analyzed the pTH-C derivative. Expression of Sox2 (Fig. $8 B, G$ ) and $R O R \alpha$ (Fig. $8 D, I$ ) was profoundly reduced in $\mathrm{Nes}{ }^{\mathrm{Cre} /+}$; Shh ${ }^{c / c}$ embryos. In contrast, Gbx2 expanded and occupied almost the entire thalamic mantle zone (Fig. $8 E, J$ ). Again, similar changes were observed in $\mathrm{Nes}{ }^{\mathrm{Cre} /+} ; \mathrm{Smo}^{c / c}$ embryos (Fig. $8 \mathrm{~K}, \mathrm{M}$ and data not shown). In $\mathrm{Olig}^{\mathrm{Cre} /+}$; $\mathrm{Smo}^{c / c}$ mice, IGL, as marked by Sox1 and neuropeptide Y (NPY) expression, was dramatically reduced in size and cell number. Sox1-positive cells in this nucleus was reduced by $\sim 70 \%\left(\mathrm{Olig}_{3}{ }^{\mathrm{Cre} /+} ; \mathrm{Smo}^{\mathrm{cl}+}: 337.5 \pm\right.$ 13.80 cells/slide, $\mathrm{Olig}^{\mathrm{Cre} /+} ; \mathrm{Smo}^{c / c}: 103 \pm 24.93$ cells/slide, $n=4$; $p=0.0002)$. This reduction likely reflects the reduction of Mash1-positive cells in the pTH-R at E12.5 (Fig. $7 M, N, R, S$ ).

In summary, decreasing Shh signaling resulted in reduction of rostral-ventral progenitor pools, pTH-R, and the rostroventral part of the $\mathrm{pTH}-\mathrm{C}$ domain, and expansion of caudodorsal progenitor pools (summarized in Fig. $9 B 1, B 2, D 1, D 2)$. This change in thalamic progenitor cell identity corresponds to alterations in postmitotic populations (Fig. $9 B 3, D 3)$. Although conditional deletion of Shh or Smo with two different Cre lines caused different degrees of reduction in Shh signal, we observed consistent changes in the progenitor cell populations as well as their postmitotic derivatives. These observations confirm the cell-intrinsic requirement of Shh signal in the specification of rostral-ventral progenitor cells in the thalamus.

\section{Relationship between Shh and Fgf signaling pathways in thalamic patterning}

A recent study by Kataoka and Shimogori (2008) reported that Fgf8 is expressed in the dorsal midline of the caudal diencephalon and that this expression domain continues to a region immediately rostral to the dorsal tip of the ZLI, suggesting that Fgf signaling may have a role in thalamic patterning. Focal in vivo electroporation of Fgf8 near the ZLI increased the size of pTH-R and its derivative, "Rim domain," although overexpression of Fgf8 did not change the expression pattern of Shh or Ptcl (Kataoka and Shimogori, 2008). These results suggest that Fgfs may play a patterning role independent of Shh signaling in the thalamus by regulating gene expression in $\mathrm{pTH}-\mathrm{R}$ progenitor cells. Alternatively, Fgf8 may be downstream of the Shh pathway and mediates part of the roles that Shh plays. In fact, Shh induces the expression of Fgf4 in early limb development (Laufer et al., 1994), and complete Shh null embryos show reduced expression of $F g f 15$ in mouse diencephalon at E8.5 (Ishibashi and McMahon, 2002). To assess the role of Shh in Fgf8 expression and Fgf signal in the thalamus, we analyzed the expression of Fgf8 and its downstream targets, Spryl and Spry2 (Liu et al., 2003) in $\mathrm{Nes}{ }^{\mathrm{Cre} /+}$; $\mathrm{R}_{2} 6^{\mathrm{SmoM} 2 /+}$ and $\mathrm{Nes}{ }^{\mathrm{Cre} /+}$; $\mathrm{Shh}^{c / \mathrm{c}}$ embryos (supplemental Fig. S4, available at www.jneurosci.org as supplemental material). We found that in both control and $\mathrm{Nes}^{\mathrm{Cre} /+}$; $R 26^{\text {SmoM2/+ }}$ embryos, Fgf8 is expressed in the dorsal midline as well as in the region immediately rostral to the dorsal part of the ZLI (supplemental Fig. S4 B, F, available at www.jneurosci.org as supplemental material). Expression of Spry1 and Spry2 was also similar in the control and SmoM2-expressing embryos (supplemental Fig. S4C, $D, G, H$, available at www.jneurosci.org as supplemental material). In addition, $\mathrm{Nes}^{\mathrm{Cre} /+}$; $\mathrm{Shh}^{c / c}$ embryos at E12.5 showed no significant reduction of expression of $F g f$, Spry1, or Spry2 (supplemental Fig. S4J-L, available at www. jneurosci.org as supplemental material) near the ZLI. These results indicate that the changes to the $\mathrm{pTH}-\mathrm{R}$ domain observed in 
$\mathrm{NeS}^{\mathrm{Cre} /+} ; \mathrm{R}^{2} 6^{\mathrm{SmoM} 2 /+}$ and $\mathrm{NeS}^{\mathrm{Cre} /+} ; \mathrm{Shh}^{\mathrm{c} / \mathrm{C}}$ embryos are not due to alterations in $F g f 8$ expression or Fgf signal.

\section{Discussion}

In this study, we used two lines of mice expressing Cre recombinase to conditionally increase or decrease Shh signaling in the thalamus, and performed in vivo electroporation to elevate the signal. When Shh signaling was globally increased using the Nes ${ }^{\mathrm{Cre}}$ allele, the entire caudal alar diencephalon, but not the surrounding brain regions including the midbrain, took on the molecular properties of the thalamus, which is consistent with previous studies using chick embryos (Vieira et al., 2005). Our current study further demonstrates that the differential Shh signal plays a crucial role not only in the specification of the most rostroventral progenitor domain of the thalamus (pTH-R) and its postmitotic derivatives, but also in the patterning within the caudodorsal thalamic progenitor domain (pTH-C) and the formation of the cortex-projecting thalamic nuclei (summarized in Fig. 9).

\section{A high Shh activity positively regulates the identity of the pTH-R domain and its postmitotic derivatives in the thalamus}

Previous studies revealed that a high Shh activity positively regulates the expression of Nkx2.2 and Sox14 in chick and mouse thalamus (Hashimoto-Torii et al., 2003; Kiecker and Lumsden, 2004; Vieira et al., 2005). We find that in the mouse, both $N k \times 2.2$ and Sox14 are expressed in the lineage of the rostral progenitor domain, pTH-R. In our present study, ectopic expression of SmoM2 resulted in increased Shh activity in thalamic progenitor cells as shown by the expression of the target genes Gli1 and Ptcl, and expansion of pTH-R markers. Postmitotic populations derived from $\mathrm{pTH}$-R progenitor cells also increased. Conversely, conditionally deleting Shh using the $\mathrm{NeS}{ }^{\mathrm{Cre}}$ allele completely abolished the expression of $\mathrm{Nkx} 2.2$ and Mash1 as well as the postmitotic markers of the pTH-R derivative. Deleting the $\mathrm{Smo}$ gene with either $\mathrm{Nes}^{\mathrm{Cre}}$ or $\mathrm{Olig}_{3}{ }^{\mathrm{Cre}}$ allele also reduced expression of pTH-R markers in progenitor cells, and resulted in corresponding decrease in its postmitotic derivative, further confirming the cell-autonomous requirement of Shh signaling in this cell lineage. Interestingly, even when SmoM2 is ectopically expressed in the entire thalamic progenitor domains either with $\mathrm{Nes}^{\mathrm{Cre}}$ or $\mathrm{Olig}^{\mathrm{Cre}}$ allele, only part of the thalamus became pTH-R; the caudodorsal part still expressed pTH-C markers (Fig. 3G, $H, R, S$ ). Because the Cre-mediated recombination seems to progress in a rostroventral to caudodorsal direction in these Cre mice, it is possible that the rostroventral part starts to express SmoM2 earlier than caudodorsal part. If SmoM2 can induce ectopic expression of pTH-R markers only before a certain stage of development, the differential onset of SmoM2 expression across the thalamic ventricular zone might explain the partial transformation of pTH-C into pTH-R. In addition, caudodorsally located thalamic progenitor cells may be less sensitive to enhanced Shh signaling than rostroventral cells. This may explain why in vivo electroporation of SmoM2 at E11.5 caused the ectopic expression of pTH-R markers only in the most rostroventral part of pTH-C (Fig. 3I,J).

However, it is also possible that establishment of pTH-R identity requires not only high Shh signaling, but also activity of other signaling molecules that are expressed in or near the ZLI or the basal plate. An intriguing possibility is Fgf8, which was recently shown to play an independent role in specifying pTH-R and its derivatives (Kataoka and Shimogori, 2008). In this current study, we found that increasing Shh activity does not enhance the expression of Fgf8 or its downstream targets, Spry1 and Spry2, and that sustained expression of Shh is not required for the expression of Fgf8, Spry1, or Spry2 in the diencephalon. Thus, if activation of both Shh and Fgf8 pathways is required for the expression of pTH-R markers, the lack of increased Fgf8 signaling in SmoM2- 


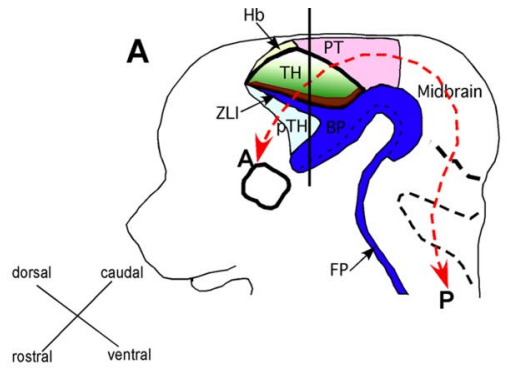

\section{Progenitor stage (E10.5-12.5)}

B1 WT

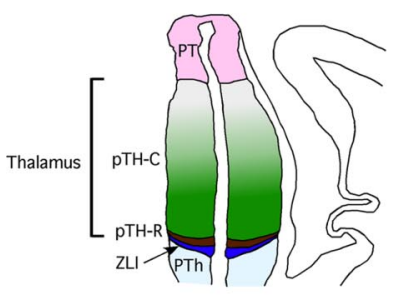

C1 SmoM2

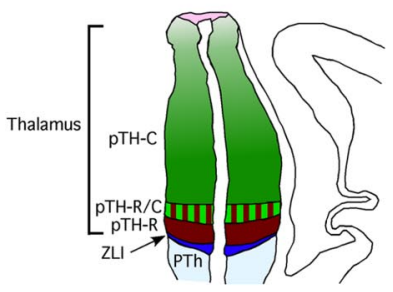

D1 Shh CKO

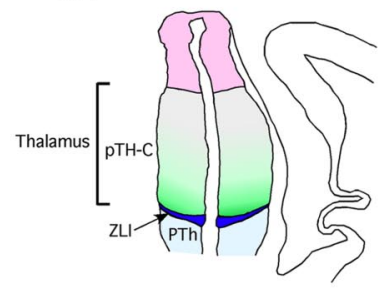

B2 $W T_{N}$

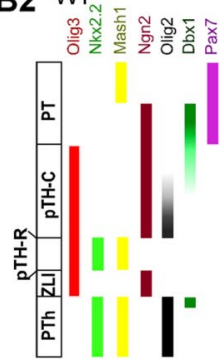

C2 SmoM2

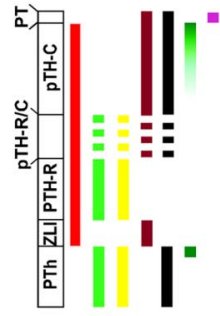

D2 Shh CKO

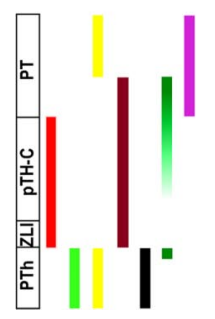

\section{Postmitotic stage (E16.5-E18.5) \\ B3 WT}
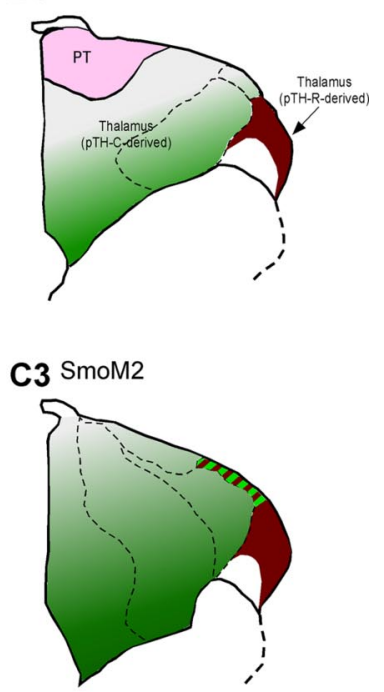

D3 Shh CKO

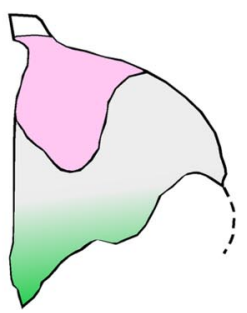

Figure 9. Summary of the current study. $A$, Side view of the embryonic thalamus. The curved anterior-posterior (A-P) axis of the brain is shown in a red dotted line. The black vertical line indicates the orientation of frontal sections used throughout this study. In a given section, the top part is more dorsal and caudal, while the bottom part is more ventral and rostral. B1, C1, D1, Schematic frontal sections showing the progenitor domains at early embryonic stages in wild type (WT), Nes ${ }^{\text {(re/+ }} ; R_{2} 6^{\text {SmoM2/+ }}$ (SmoM2), and $\mathrm{NeS}^{\mathrm{Cre} /+}$; Shh ${ }^{c / c}$ (Shh cko) mice. Color gradients within PTH-C indicate the graded differential patterns of gene expression within this domain, which are altered when Shh signal is increased (C1) or decreased (D1). B2, C2, D2, Differential gene expression patterns in each genotype. $B 3, C 3, D 3$, Schematic frontal sections indicating the postmitotic thalamus at late embryonic stages. The graded color of $\mathrm{pTH}-\mathrm{C}-\mathrm{derived}$ thalamus corresponds to the overall lineage relationship with the progenitor domains shown in $\mathbf{B 1}, \mathbf{C}$, and $\mathbf{D}$ 1. Alterations in SmoM2-expressing mice and Shh cko mice indicate that the changes in the mantle zone are consistent with those observed in progenitor cells.

expressing thalamus may account for the limited expansion of pTH-R domain. However, since Fgf8 is expressed only near the dorsal tip of the ZLI, not in the entire ZLI, it is unclear whether endogenous Fgf8 signaling is required for all the progenitor cells in the pTH-R domain. Further studies are needed to identify the collaboration of signaling pathways responsible for the pTH-R identity.

How does Shh activity control the patterning of the pTH-C domain and the cortex-projecting thalamic nuclei? Compared with the contribution of Shh signaling to the pTH-R lineage, understanding the role of Shh activity in the caudodorsal progenitor domain, $\mathrm{pTH}-\mathrm{C}$, is more challenging. The $\mathrm{pTH}-\mathrm{C}$ domain contains highly heterogeneous progenitor populations characterized by graded expression of transcription factors such as Olig2 or Dbx1. In addition, the many dozens of distinct, cortexprojecting thalamic nuclei that are generated from this domain are not always identifiable by marker gene expression alone, and the use of cytoarchitectural features to discriminate individual nuclei is also limited. Because of these difficulties, how these nuclei are patterned and specified during embryogenesis is not well understood. We approached this question by first identifying and characterizing the molecular differences within $\mathrm{pTH}-\mathrm{C}$, which we found contributes to all the cortex-projecting thalamic nuclei. This analysis indicated that heterogeneity within this progenitor domain may contribute to the specification of multiple thalamic nuclei (Vue et al., 2007). Our current study now shows for the first time that the level of Shh signaling controls the identity of $\mathrm{pTH}-\mathrm{C}$ progenitor cells, and that this in turn regulates the sizes and identity of postmitotic thalamic nuclei.

We showed that high Shh signaling positively regulates the expression of Olig2 and Pdlim 3 in the rostral part of pTH-C. Conversely, conditional deletion of Shh or Smo with the Nes $\mathrm{Crel}^{+}$allele diminishes the expression of Olig2 in pTH-C, while it expands the expression of Dbx1, a marker for caudodorsal pTH-C. In addition, increasing or decreasing Shh signaling altered regional patterns of gene expression in the postmitotic thalamus in a way that was consistent with the changes in progenitor cells. Among the postmitotic markers we analyzed, $R O R \alpha$ and Sox 2 are expressed more strongly in the nuclei that are derived from the rostroventral part of the pTH-C domain. In SmoM2-expressing embryos, Sox 2 and $R O R \alpha$ are expanded to occupy the entire rostrocaudal extent of the postmitotic thalamus, consistent with the expanded expression of Olig2 and Pdlim3. Shh and Smo knock-out brains show the opposite overall changes. These results clearly demonstrate that differential Shh activity controls not only the identity of pTH-C progenitor cells but also the spatial organization of the postmitotic thalamic nuclei. When SmoM2 was conditionally expressed using the $\mathrm{Olig} 3^{\mathrm{Cre}}$ allele, the dLG nucleus, which is derived from the rostroventral part of pTH-C, was indeed expanded based on anterograde axon tracing from the retina. This confirms an intrinsic role of Shh signaling in the formation of a specific thalamic nucleus that projects to the cortex. 
A recent study by Szabó et al. (2009) used Foxb1-Cre mice to broadly delete Shh in the developing CNS and found grossly abnormal thalamus and aberrant expression of postmitotic markers at E18.5. They also found that markers broadly expressed in thalamic progenitor cells such as Olig3 and Ngn2 were still expressed in the mutant mice. Based on these observations, they argued that Shh is mainly required in postmitotic cells in the thalamus to promote migration and aggregation into nuclei as well as axonal extension to the cortex rather than neuronal fate acquisition. In contrast, our current study strongly indicates that Shh acts primarily on thalamic progenitor cells. First, markers that are normally expressed in graded manners within the pTH-C domain such as Olig2, Pdlim3, and Dbxl were significantly affected when Shh activity was altered. The observed alteration of the postmitotic thalamus reflected the changes in the expression of these progenitor cell markers. Second, direct target genes of Shh signaling such as Gli1 and Ptcl were expressed only in thalamic progenitors, even though SmoM2 was mis-expressed in both progenitor and postmitotic cells after Cre-mediated recombination. Gli1 and Ptc1 expression was diminished in progenitor cells when the Shh signal was perturbed. Based on these observations, we conclude that Shh acts primarily on thalamic progenitor cells, and the graded Shh signal is essential to confer thalamic progenitors with position-dependent molecular heterogeneity, which forms a basis for generating a diverse set of postmitotic thalamic nuclei. Although the study by Szabó et al. (2009) and our current study used different Cre lines to delete the Shh gene, both observed the complete absence of the pTH-R marker Nkx2.2. Given the early and broad deletion of Shh in their conditional mutant mice, it is also likely that expression of graded pTH-C markers were altered as well. Thus, we think the different conclusions of the two studies have resulted from different interpretations of potentially similar data. Although we cannot completely rule out the possibility that Shh plays some role in postmitotic differentiation of thalamic neurons such as migration or axonal projection, demonstration of such roles would require different sets of experiments where Shh signal is altered in postmitotic cell-specific, conditional gene targeting in mice, or by in vitro assays that exclude the involvement of progenitor cells.

If Shh signaling regulates the expression of transcription factors in thalamic progenitor cells, then do these transcription factors play a functional role in specifying the formation of the various nuclei along the rostral-caudal and dorsal-ventral axes? We found by overexpression experiments that Olig2 can induce rostroventral thalamic nucleus marker Sox 2 in the dorsal-caudal thalamus. However, Olig2 mutant mice show normal expression of Sox2, indicating that other factors are also involved. Further studies are needed to identify such genes and to test the individual roles of each of the other transcription factors.

In conclusion, we believe that this study provides a unique perspective in neural development in several respects. First, we showed how Shh patterns a single developmental unit in the embryonic diencephalon ( $\mathrm{pTH}-\mathrm{C}$ ) to generate multiple neuronal groups that are grossly arranged in similar rostrocaudal and dorsoventral locations to those of their precursor cells. This feature makes the developing thalamus a good model system to study how early patterning events contribute to the specification of multiple neuronal fates that share some basic properties such as the projection to the neocortex and use of glutamate as neurotransmitter. Second, connectivity and functions of thalamocortical projections are relatively well understood, especially for those from the principal sensory thalamic nuclei. In this study, by using Olig $3^{\mathrm{Cre}}$ mice, we were able to generate postnatally viable mice in which Shh signaling was specifically enhanced or reduced in the thalamus without primarily affecting the development of the cortex or brain regions along the thalamocortical pathway. These mice will allow us to examine at the systems level the neuroanatomical and functional consequences of altering the early patterning of the thalamus.

\section{References}

Agren M, Kogerman P, Kleman MI, Wessling M, Toftgård R (2004) Expression of the PTCH1 tumor suppressor gene is regulated by alternative promoters and a single functional Gli-binding site. Gene 330:101-114.

Bai CB, Stephen D, Joyner AL (2004) All mouse ventral spinal cord patterning by hedgehog is Gli dependent and involves an activator function of Gli3. Dev Cell 6:103-115.

Briscoe J, Novitch BG (2008) Regulatory pathways linking progenitor patterning, cell fates and neurogenesis in the ventral neural tube. Philos Trans R Soc Lond B Biol Sci 363:57-70.

Dessaud E, Yang LL, Hill K, Cox B, Ulloa F, Ribeiro A, Mynett A, Novitch BG, Briscoe J (2007) Interpretation of the sonic hedgehog morphogen gradient by a temporal adaptation mechanism. Nature 450:717-720.

Fuccillo M, Joyner AL, Fishell G (2006) Morphogen to mitogen: the multiple roles of hedgehog signalling in vertebrate neural development. Nat Rev Neurosci 7:772-783.

Gray PA, Fu H, Luo P, Zhao Q, Yu J, Ferrari A, Tenzen T, Yuk DI, Tsung EF, Cai Z, Alberta JA, Cheng LP, Liu Y, Stenman JM, Valerius MT, Billings N, Kim HA, Greenberg ME, McMahon AP, Rowitch DH, et al. (2004) Mouse brain organization revealed through direct genome-scale TF expression analysis. Science 306:2255-2257.

Hashimoto-Torii K, Motoyama J, Hui CC, Kuroiwa A, Nakafuku M, Shimamura K (2003) Differential activities of Sonic hedgehog mediated by Gli transcription factors define distinct neuronal subtypes in the dorsal thalamus. Mech Dev 120:1097-1111.

Ishibashi M, McMahon AP (2002) A sonic hedgehog-dependent signaling relay regulates growth of diencephalic and mesencephalic primordia in the early mouse embryo. Development 129:4807-4819.

Jeong J, Mao J, Tenzen T, Kottmann AH, McMahon AP (2004) Hedgehog signaling in the neural crest cells regulates the patterning and growth of facial primordia. Genes Dev 18:937-951.

Jones EG (2007) The thalamus. Cambridge UP: New York.

Kataoka A, Shimogori T (2008) Fgf8 controls regional identity in the developing thalamus. Development 135:2873-2881.

Kaufman MH (1992) Atlas of mouse development. San Diego: Academic.

Kiecker C, Lumsden A (2004) Hedgehog signaling from the ZLI regulates diencephalic regional identity. Nat Neurosci 7:1242-1249.

Laufer E, Nelson CE, Johnson RL, Morgan BA, Tabin C (1994) Sonic hedgehog and Fgf- 4 act through a signaling cascade and feedback loop to integrate growth and patterning of the developing limb bud. Cell 79:993-1003.

Lewis PM, Dunn MP, McMahon JA, Logan M, Martin JF, St-Jacques B, McMahon AP (2001) Cholesterol modification of sonic hedgehog is required for long-range signaling activity and effective modulation of signaling by Ptc1. Cell 105:599-612.

Liu A, Li JY, Bromleigh C, Lao Z, Niswander LA, Joyner AL (2003) FGF17b and FGF18 have different midbrain regulatory properties from FGF8b or activated FGF receptors. Development 130:6175-6185.

Long F, Zhang XM, Karp S, Yang Y, McMahon AP (2001) Genetic manipulation of hedgehog signaling in the endochondral skeleton reveals a direct role in the regulation of chondrocyte proliferation. Development 128:5099-5108.

Machold R, Hayashi S, Rutlin M, Muzumdar MD, Nery S, Corbin JG, GritliLinde A, Dellovade T, Porter JA, Rubin LL, Dudek H, McMahon AP, Fishell G (2003) Sonic hedgehog is required for progenitor cell maintenance in telencephalic stem cell niches. Neuron 39:937-950.

Mukouyama YS, Deneen B, Lukaszewicz A, Novitch BG, Wichterle H, Jessell TM, Anderson DJ (2006) Olig2 + neuroepithelial motoneuron progenitors are not multipotent stem cells in vivo. Proc Natl Acad Sci U S A 103:1551-1556.

Nakagawa Y, O’Leary DD (2003) Dynamic patterned expression of orphan 
nuclear receptor genes RORalpha and RORbeta in developing mouse forebrain. Dev Neurosci 25:234-244.

Puelles L, Rubenstein JL (1993) Expression patterns of homeobox and other putative regulatory genes in the embryonic mouse forebrain suggest a neuromeric organization. Trends Neurosci 16:472-479.

Puelles L, Rubenstein JL (2003) Forebrain gene expression domains and the evolving prosomeric model. Trends Neurosci 26:469-476.

Saito T, Nakatsuji N (2001) Efficient gene transfer into the embryonic mouse brain using in vivo electroporation. Dev Biol 240:237-246.

Shimamura K, Hartigan DJ, Martinez S, Puelles L, Rubenstein JL (1995) Longitudinal organization of the anterior neural plate and neural tube. Development 121:3923-3933.

Srinivas S, Watanabe T, Lin CS, William CM, Tanabe Y, Jessell TM, Costantini F (2001) Cre reporter strains produced by targeted insertion of EYFP and ECFP into the ROSA26 locus. BMC Dev Biol 1:4.
Szabó NE, Zhao T, Zhou X, Alvarez-Bolado G (2009) The role of Sonic hedgehog of neural origin in thalamic differentiation in the mouse. J Neurosci 29:2453-2466.

Tronche F, Kellendonk C, Kretz O, Gass P, Anlag K, Orban PC, Bock R, Klein R, Schütz G (1999) Disruption of the glucocorticoid receptor gene in the nervous system results in reduced anxiety. Nat Genet 23:99-103.

Vieira C, Garda AL, Shimamura K, Martinez S (2005) Thalamic development induced by Shh in the chick embryo. Dev Biol 284:351-363.

Vue TY, Aaker J, Taniguchi A, Kazemzadeh C, Skidmore JM, Martin DM, Martin JF, Treier M, Nakagawa Y (2007) Characterization of progenitor domains in the developing mouse thalamus. J Comp Neurol 505:73-91.

Xie J, Murone M, Luoh SM, Ryan A, Gu Q, Zhang C, Bonifas JM, Lam CW, Hynes M, Goddard A, Rosenthal A, Epstein EH Jr, de Sauvage FJ (1998) Activating Smoothened mutations in sporadic basal-cell carcinoma. Nature 391:90-92. 